\title{
Shunting Strategy for Placing-In and Taking-Out Wagons on Branch-Shaped Freight Operation Network with Simulation Method
}

\author{
Bing Li (D), Wenyao Qu, Hua Xuan (D), Chunqiu Xu, Xueyuan Wang, and Yifei Geng \\ School of Management Engineering, Zhengzhou University, Zhengzhou 450001, Henan, China \\ Correspondence should be addressed to Bing Li; lbing@zzu.edu.cn
}

Received 10 May 2019; Revised 3 December 2019; Accepted 26 December 2019; Published 22 January 2020

Academic Editor: Eneko Osaba

Copyright ( 2020 Bing Li et al. This is an open access article distributed under the Creative Commons Attribution License, which permits unrestricted use, distribution, and reproduction in any medium, provided the original work is properly cited.

\begin{abstract}
Because of China's vast territory, large population, and huge demand for bulk materials, the railway transportation mode has always received considerable attention. For long-haul transportation, railway transportation can provide a scheduled-based transport plan, all-weather transport service, and cheaper carry instead of other transport modes. The study described in this paper attempts to develop a simulation platform for optimizing the placing-in and taking-out wagons system on the branchshaped freight operation network (PTWS-BSFON). The operation process of PTWS is thoroughly analyzed from three aspects of decoupling-coupling wagons subsystem, placing-in local wagons subsystem, and taking-out local wagons subsystem. And then the simulation platform encompassing two typically shunting modes of PTWS are developed by Arena software. Under scenarios of PTWS-SO and PTWS-SSMS, the hierarchical structure of the shunting strategies is, respectively, outlined in the simulation platform. Finally, the shunting strategies based on the simulation platform are carried out by concrete examples, which prove the rationality of the methodology in applying different strategies and enhancing the performances of the PTWSBSFON.
\end{abstract}

\section{Introduction}

The growth of China's economy has led to a constant increasing for bulk commodity and long-haul transportation. Railway transport with strictly schedule-based operation modes mainly delivery bulk commodity and undertake main-line transport task. So railway transport has received considerable attention due to the specific status of railway transport in China. Railway freight terminal is well recognized as one of the most important and critical elements for enhancing the competitiveness of the railway freight transportation chain. Therefore, the performance of a railway freight terminal is crucial for the transportation chain effectiveness and must to be closely optimized.

Taking-out and placing-in shunting of wagons is an important work for the large railway freight terminal. Their efficiency is directly related to the wagons turnover, the goods delivery, and the completion of the transport production quota. The operators of a railway terminal must think how to arrange reasonably placing-in and taking-out shunting of wagons. In the whole process of placing-in and taking-out shunting, the local wagons will be decoupled from inbound trains, delivered to relevant freight operation sites for loading or unloading, retrieved back from the freight operation site, and couple onto outbound trains. Depending on the layout of freight operation sites in a railway terminal, we can divide them into two types: radial freight operation network (RFON) and branch-shaped freight operation network (BSFON). In a radial freight operation network, after the shunting engine has sent a wagon group to one freight operation site, it must go back to the railway station before running on to the next site. For a branch-shaped freight operation network, the shunting engine does not need to return to the railway station before placing-in another wagon group. In this paper, we discuss the branch-shaped freight operation network. This paper 
sets out to develop a simulation approach for evaluating and optimizing the placing-in and taking-out wagons system on the branch-shaped freight operation network (PTWSBSFON) on the basis of economic considerations. The simulation methods for placing-in and taking-out wagons system focus on minimizing shunting cost.

Simulation is an imitation of a real-world process and draws inferences regarding the characteristics of the real system. The goal of system simulation techniques is designing a model for a real system which allows users to observe the approximate behavior of a real system. System simulation can identify the system bottlenecks and assist future improvements, creating an optimal condition between the inputs and outputs. In placing-in and taking-out wagons system, simulation can be used as a tool for designing different scenarios with the aim of mitigating the negative effects of inefficient operation existing in the system.

The main reason to this study motivation is that there are high operation costs for loading, unloading, shunting work, and so on in PTWS-BSFON. Therefore, this study focuses to answer this high operation costs reduction for the placing-in and taking-out wagons system considering all possible strategies described in this study. The PTWS represents a significant source of delay for wagons detention at a railway terminal, which affects the railway terminal's efficiency and their reputation. Our simulation model assists PTWSBSFON in improving the efficiency of technical operations, raising shunting engine productivity, and speeding up wagon circulation.

Overall, the objectives of this research are twofold. The first objective is to develop a novel simulation platform in PTWS-BSFON. The simulation platform encompassing the two typically shunting modes of PTWS-BSFON are developed by Arena software, and the hierarchical structure of shunting strategies based on the simulation module is outlined. The second objective is to apply the output from the simulation program results to assessing the impacts produced by the adoption of different policies and improving the organization scheme for operators.

The remainder of this paper is organized as follows. Section 2 of this paper discusses related earlier research efforts. Section 3 is devoted to the description and analysis of the PTWS-BSFON. In Sections 4 and 5, we develop the shunting strategies of PTWS-BSFON and build the simulation-based optimization framework by Arena software. The experiments are described in Section 6, and the effectiveness of the proposed shunting strategies is shown from the performance results. The last section concludes with a summary of current work and extensions.

\section{Literature Review}

In this section, we review the relevant literatures about placing-in and taking-out shunting of wagons problems. Literature review indicates that they can be divided into three groups, that is, the operation mechanism of PTWS, mathematical methodology for PTWS, and simulation approach for PTWS. The focus of our literature review will primarily be on the model and exact approach since it is also what we have taken in this paper.

2.1. Overview of Previous Research. Some literatures on PTWS were addressed from the viewpoint of the operation mechanism. Dahle et al. [1] considered the compensation mechanism in pickup and delivery operation with time window. Urrutia and De Werra [2] considered both limited and unlimited capacity stacks and allowed some transformations on the delivery route. Adamo et al. [3] studied the optimization of delivery routes and speeds of goods with time window constraints. Haddad et al. [4] studied the problem of batch pickup and delivery of goods. Danloup et al. [5] addressed the problem of taking and delivering goods with transhipment. Wang et al. [6] studied the cooperative green delivery problem aiming at minimizing carbon emissions and proposed a precise solution strategy based on cooperative game theory to complete the compensation and profit allocation scheme of partners. Zhu and Sheu [7] described the problem of synchronous delivery of goods with stochastic demand and proposed a new delivery strategy. The advantages of the proposed cargo delivery strategy were verified by comparing it with the strategy of not allowing vehicles to cooperate with each other. Györgyi and Kis [8] studied a dynamic and stochastic pickup and delivery problem. They demonstrated that the cost structure of the problem permits an effective solution method without generating multiple scenarios.

In recent years, many researches considered the PTWS using mathematical model methodology and heuristic algorithm from different perspectives. In order to minimize the total travel distance as well as the total handling cost at the warehouse, Li et al. [9] proposed a branch-and-priceand-cut algorithm based on a strong set-partitioning model and designed an ad-hoc label-setting algorithm. Ghilas et al. [10] proposed a branch and bound algorithm for cargo pickup and delivery with time windows. Capelle et al. [11] studied the location-routing problem considering cargo delivery operations, constructed the integer programming model of the problem, and proposed a column generation algorithm. Lu and Yang [12] proposed a hybrid approach called the iterative logistics solution planner (ILSP) for enhancing the quality of delivery and pickup operation in logistics. Li et al. [13] focused on particular vehicle assignment problems arising when performing loading or unloading operations, developed a bilevel programming model, and proposed the alternating algorithm by updating predicted control parameters. And then, Li et al. [14] deal with multistage heterogeneous fleet scheduling with fleet sizing decisions, set up a mixed integer programming model, and proposed a hybrid simulated annealing algorithm. Giovanni et al. [15] proposed a two-level local search heuristic to solve pickup and delivery problem with multiple attributes. Their approach can not only assign orders to vehicles but also optimize the route service sequences. Goeke [16] developed a granular tabu search with a policy to determine the amount of energy recharged to plan routes in order to satisfy requests. Dragomir et al. [17] introduced 
a new type of problem scenario combining various attributes: a pickup and delivery problem with multiple regions, multiple depots, and multiple transportation modes. A time-indexed integer linear programming model was proposed by Wang and Chen [18] for the delivery problem of truck scheduling for a supplier. Ulmer et al. [19] explored same-day delivery routing and particularly how same-day delivery vehicles can better integrate dynamic requests into delivery routes by taking advantage of preemptive depot returns. Benavent et al. [20] discussed a pickup-and-delivery single-vehicle routing problem where there is susceptibility to uncertainty in customer requests. Bettinelli et al. [21] considered customers and facilities with time windows and proposed branch-and-cut-and-price algorithm to solve the multitrip separate pickup and delivery problem. For the pickup and delivery problem with time windows and last-in-first-out loading, Alyasiry et al. [22] proposed a novel exact approach based on fragments-a series of pickup and delivery requests starting and ending with an empty vehicle. For the pickup and delivery problem with transfers, Mahmoudi et al. [23] developed a continuous time approximation approach using cumulative arrival, departure, and on-board count diagrams to effectively assess the performance of the system and dynamically constrict the search space. Huang et al. [24] proposed a hybrid heuristic algorithm combined with modified Clarke-Wright saving algorithm and tabu search to solve a complex electric logistics vehicle routing problem.

Simulation methodology was adopted for evaluating and improving the PTWS in some literatures. Jaehn et al. [25] evaluated performances of their heuristic solution in simulations with different scenarios and provide their worst-case performance guarantee when studying shunting operations problem at flat yards. Kieu et al. [26] considered important interactions between buses and their schedules and formulated several simulation models of bus routes have been proposed in the literature, including cellularautomata, bus-following, and traffic-following models to make transit operators both better understand the dynamics of bus routes and facilitate better policymaking. Zhu et al. [27] proposed a simulation-based quantitative analysis and an evaluation method to solve the comparison problem of water-water transhipment coal terminals. Liu et al. [28] used the simulation method to analyze connection of indoor occupants with the energy consumption of heating, ventilation, and air-conditioning (HVAC) in airport terminals which are characterized by passenger flow. Archetti et al. [29] presented the results of a simulation study aimed at assessing an on-demand transportation system. Lam et al. [30] developed a large simulation platform to study the problem of public transportation systems with autonomous vehicles so that analysis and visualization of transportation technologies were allowed. Deja et al. [31] combined the simulation method with an algorithm to study quayside transport and storage yard operations in container terminals. Florin et al. [32] used the discrete simulation model to evaluate the transit capacity of port shunting yards.
2.2. Contribution. Specifically, the novelty of this paper is as follows:

(1) The operators of a railway terminal prefer to explore whether new management methodologies can improve the PTWS performance before investing in new equipment or enlarging the size of the PTWS. This paper aims to analyze the performance of PTWS-BSFON using simulation models and obtain the efficient organization scheme of PTWS-BSFON working for operators. Instead of obscure and inaccessible mathematical methodology, computerbased simulation approach can intuitively provide the decision-makers with the help they need in creating the strategies for development.

(2) We identify the decoupling-coupling local wagons in a railway station, delivery the local wagons group from the railway station to the freight operation site, and retrieve the local wagons group from the freight operation site to railway station. Based on that, the whole operation process of PTWS is thoroughly analyzed from three aspects of decoupling-coupling local wagons subsystem, placing-in local wagons subsystem, and taking-out local wagons subsystem.

(3) We develop the simulation platform encompassing the two typically shunting modes of PTWS by Arena software. And then, the hierarchical structure of this simulation module is, respectively, outlined in the simulation platform. Under scenarios of PTWS-SO, we provide the eight shunting strategies. Under scenarios of PTWS-SSMS, we present two different span-shift-moving strategies and generate sixteen hybrid shunting strategies.

(4) We test the performance of the simulation platform by applying for many instances. According to our results, our simulation platform has a better performance on assessing the impacts produced by the adoption of different shunting strategies and providing the efficient organization scheme of PTWS for operators.

\section{Problem Description and Analysis}

This section describes the whole processes of placing-in and taking-out wagons on branch-shaped freight operation networks. We make a brief overview of some foundational concepts and analysis of the problem.

3.1. Problem Description. Placing-in and taking-out shunting of wagons is an important and complicated job for a large railway terminal. Many impact factors such as arrival time of inbound trains, departure time of outbound trains, local wagons amount, loading or unloading time of local wagons in freight operation sites, and trip time of shunting engine for placing-in and taking-out wagons between freight operation sites should be taken into consideration. Railway terminal is consisted of a railway station and freight 
operation sites. The railway station is the basic unit for transport production. Freight operation sites are the cargo loading and unloading locations of the railway station. Local wagons are often transferred between railway station and freight operation site when placing-in and taking-out operations begin. So the railway terminal mainly includes railway station and freight operation sites. Understanding the structure of the railway terminal, the flow among them, and operation scenarios within them is very necessary.

Railway station has some yards where arriving operation, departure operation, and shunting operation for inbound train and outbound train are carried out. Receiving departure yard, shunting yard, and locomotive depot are usually set up in rail terminals in order to finish these technical operations. The inbound trains first enter into the receiving yard and then into the shunting yard for placing-in and taking-out of wagons. Finally, the local wagons taken out from freight operation sites will go back to the departure yard and then depart from the railway station.

The freight operation network is composed of freight operation sites where local wagons can be loaded or unloaded. Here, we discuss the layout of branch-shaped freight operation sites. There are two types of local wagons in the freight operation site, namely, empty wagon group and heavy wagon group. When the empty wagon group arrives at the relevant freight operation site, they will be detached and put down for loading operation. When the heavy wagon group arrives at the relevant freight operation site, they will begin to unload. These local wagons should be retrieved after their tasks finish and pull back to the railway station in accordance with the taking-out plan.

On the basis of the analysis of placing-in and taking-out wagons system on the branch-shaped freight operation network and gathering from an in-depth literature review, we identify the system to need three main processes: the decoupling and coupling process of local wagons from the train, delivery the wagons group to freight operation sites, and pick up the wagons group from the freight operation sites. So PTWS-BSFON can be divided into three parts, e.g., decoupling-coupling wagons subsystem (DCWS), placing-in wagons subsystem (VWLS), and taking-out wagons subsystem (TOWS).

\subsection{Understanding the Operation Process of Decoupling-} Coupling Local Wagons Subsystem. The hubs of placing-in and taking-out local wagons system are the railway station. Local wagons which need to be dispatched to the freight sites for loading or unloading are decoupled from inbound trains in the railway station. Meanwhile, these local wagons which are taken out from the freight sites after loading or unloading are coupled into new outbound trains in the railway station. The railway station consists of an arrival yard, a departure yard, and a shunting yard that has a classification bowl with a sophisticated braking system. The arrival yard and shunting yard are often connected by a hump, and a switching system directs the wagons to their assigned classification bowl tracks of the shunting yard.

The inbound freight trains arriving to a railway station are parked on the arrival yard where local wagons are decoupled and inspected. These local wagons are then rolled into the shunting yard where they are sorted into their assigned classification bowl tracks. When the accumulation number of local wagons in the shunting yard reaches a specified size, the placing-in operations are carried out. A shunting engine is sent from the locomotive depot, and all local wagons are coupled. Then, these wagons are dispatched to relevant operation sites of the freight operation network for loading or unloading according to a certain rule. After the loading and unloading tasks are finished, the wagons are then taken out in a certain order and pulled back to the shunting yard where they are sorted into new outbound trains. Finally, outbound trains must departure from the departure yard. The process block diagram illustrating the operation process of local wagons receiving-departure subsystem in a railway station in this research is shown in Figure 1.

\subsection{Understanding the Operation Process of Placing-In} Wagon Subsystem. On arrival, the local wagons are decoupled from inbound trains and repositioned to the shunting yard. When the accumulation number of local wagons reaches a specified scale, the operation of placing-in wagons is carried out. A shunting engine is sent from the engine depot, and all local wagons are coupled by the shunting engine. Shunting engine dispatches them to relevant freight operation sites one by one. After arriving at the freight operation site, the wagon group is detached and put in the right place. In the freight operation sites, the wagon group is arranged to load or unload. And then, the shunting engine goes to another freight operation site. In the process of placing-in wagons, it is very important to assign reasonably the sequence of freight operation sites. In this research, the process block diagram illustrating the operation process of placing-in wagons from railway station to freight operation sites is shown in Figure 2.

\subsection{Understanding the Operation Process of Taking-Out} Wagon Subsystem. After all local wagons are dispatched to relevant freight operation sites, placing-in operation is finished and taking-out operation begins. The shunting engine will return to these freight operation sites and retrieve the wagon group one by one. After shunting engine arrives at the freight operation site, the engine attaches the wagon group which have finished the loading or unloading. And then, the shunting engine goes to another freight operation site. When all wagon group are retrieved from freight operation sites, the shunting engine pulls them back to the shunting yard where they are sorted into new outbound trains. In the process of taking-out wagons, the reasonable assignment of freight operation sites sequence is the key. In this research, the process block diagram illustrating the operation process of taking-out wagons from freight operation sites to the railway station is shown in Figure 3.

\section{Simulation-Based Optimization Framework}

The aims of this project are to implement a simulation environment and assess the impacts produced by the adoption of different shunting policies so that the 


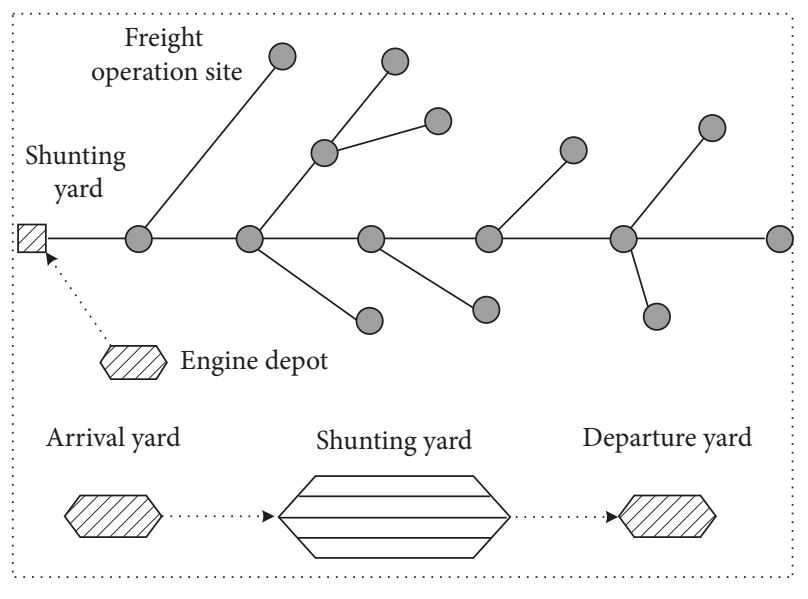

Branch-shaped freight operation sites

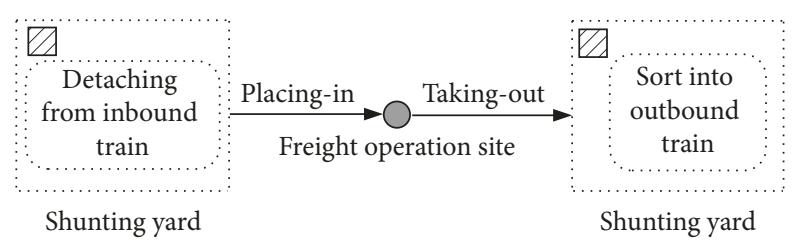

\begin{tabular}{|c|c|c|}
\hline Work location & Work item & Work time \\
\hline Arrival yard & Inbound train arrival & \\
\hline Engine depot & Dispatching shunt engine & \\
\hline Shunting yard & Attaching wagon & \\
\hline Railway special line & Placing-in wagon & \\
\hline Freight operation site & Loading and unloading & 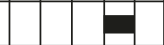 \\
\hline Railway special line & Taking-out wagon & \\
\hline Shunting yard & Sort into outbound train & \\
\hline Departure yard & Outbound train departure & \\
\hline
\end{tabular}

FIgURE 1: Illustration of the operation process for decouplingcoupling local wagons subsystem.

performances of PTWS-BSFON are enhanced. To achieve this objective, the project needs to encompass all the scenarios of PTWS-BSFON. The two scenarios are comprehensively considered and, respectively, model the simulation, e.g., placing-in and taking-out wagons system with single operation (PTWS-SO) and placing-in and taking-out wagons system with span-shift-moving shunting (PTWS-SSMS). The models of PTWS-BSFON are developed by the Arena simulation platform. After that, we analyzed the output from the simulation program results and applied the analysis to produce a group of alternatives. We ranked these alternatives to choose the best alternative to improve the efficiency of our system to get a better service quality. For expositional reasons, we introduce some notations to represent the branch-shaped freight operation network. Let $G(O, N, E)$ represent the freight operation network, where $O$ is the railway station, $N$ denotes a set of freight operation sites, and $E$ represents the set of the movement of local wagons between a pair of freight operation sites or between railway station and freight operation site. Define $N=\{1, \ldots, A\}$, where $A$ is the number of freight operation sites. We define $t_{i j}$ as the travel time from $i$ to $j, i, j \in N$.
Freight operation site of placing-in wagon

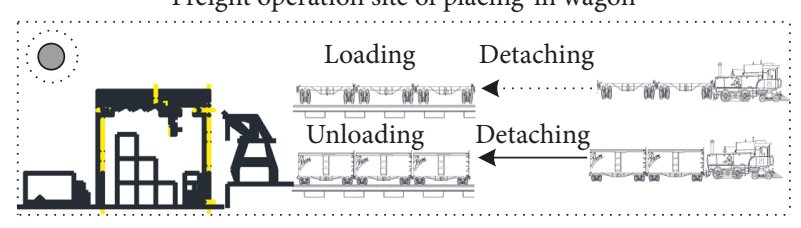

Freight operation site of placing-in wagons

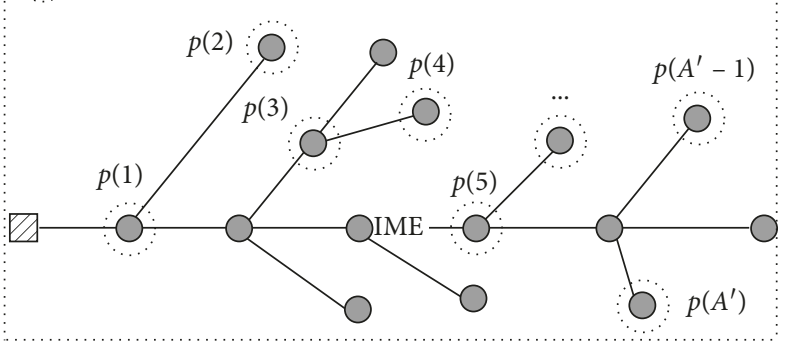

Placing-in wagons sequence $\left\{p(\delta), \delta=1, \ldots, A^{\prime}\right\}$

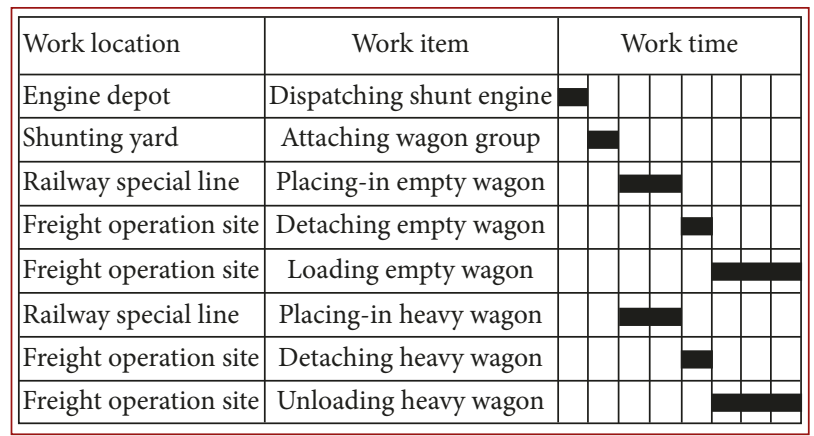

FIGURE 2: Illustration of the operation process for placing-in wagon subsystem.

4.1. Simulating PTWS-SO. Under scenarios of PTWS-SO, all local wagons belong to single operation wagon. That is to say, the loading or unloading can only be carried out for once after the local wagon group is detached at relevant freight operation site. The local wagon for double freight operations which is moved from one site to another site for second loading or unloading cannot be allowed. In the section, we discuss the problem of simulating PTWS-SO with eight shunting strategies which are abbreviated, respectively, as ATT-ECT, ADT-ECT, RTT-ECT, RDT-ECT, ATT-NL\&CT, ADT-NL\&CT, RTT-NL\&CT, and RDTNL\&CT.

4.1.1. Selecting Operation Sites Sequence Based on Minimizing Absolute Travel Time and Earliest Completion Time. In this section, we will develop the procedure of the simulation module for the shunting strategy of placing-in site sequence and taking-out site sequence based on minimizing absolute travel time and earliest completion time. This shunting strategy is abbreviated as ATT-ECT in order to facilitate representing the problem.

Step 1 (generating placing-in freight operation sites sets). According to the destination freight operation site of local wagons, we choice the relevant operation sites 


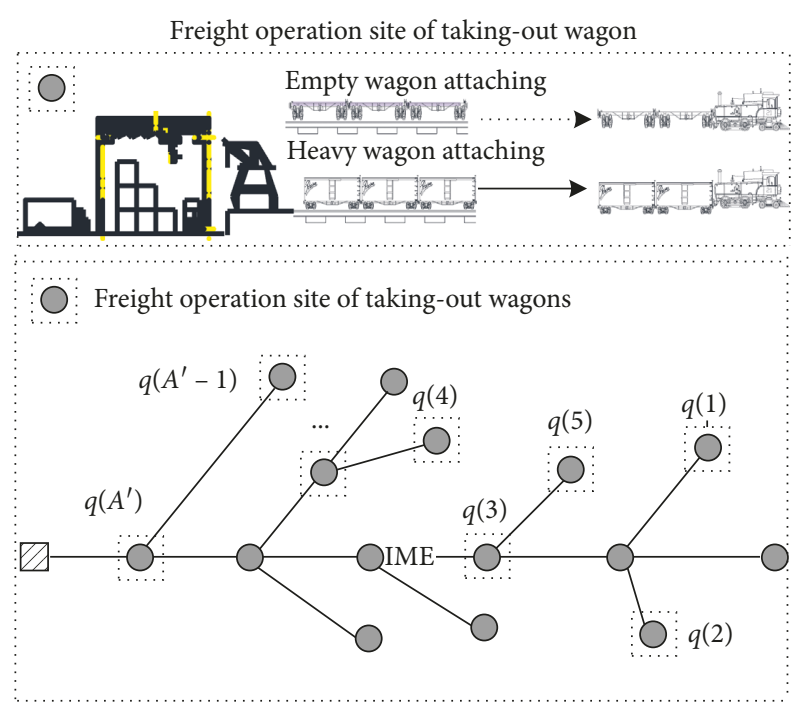

Taking-out wagons sequence $\left\{q\left(\delta^{\prime}\right), \delta^{\prime}=1, \ldots, A^{\prime}\right\}$

\begin{tabular}{|c|c|c|}
\hline Work location & Work item & Work time \\
\hline Freight operation site & Attaching wagon group & \\
\hline Railway special line & Taking-out empty wagon & \\
\hline Shunting yard & Detaching empty wagon & \\
\hline Railway special line & Taking-out heavy wagon & \\
\hline Shunting yard & Detaching heavy wagon & \\
\hline
\end{tabular}

FIGURE 3: Illustration of the operation process for taking-out wagon subsystem.

from the set of freight operation sites $N$. So the set of placing-in freight operation sites $S$ can be obtained. Define $S=\left\{1, \ldots, A^{\prime}\right\}$, where $A^{\prime}$ is the total number of placing-in freight operation sites.

Step 2 (determining placing-in freight operation site selection sequence). We define the absolute travel time as $t_{o j}$. It represents the travel time from railway station $o$ to any freight operation site $j \in S$. And then, we count the absolute travel time $t_{o j}$ and rank the freight operation sites in ascending order. So the placing-in freight operation site selection sequence $P^{\text {placing-in }}$ can be obtained. We denote $\delta$ as the serial number of the placing-in freight operation site. Thus, $P^{\text {placing-in }}$ can be expressed as

$$
\begin{aligned}
P^{\text {placing-in }}=\{p(\delta) \mid p(\delta) & \left.=p, p \in S, \delta=1, \ldots, A^{\prime}\right\}, \\
& t_{o p(1)} \leq t_{o p(2)} \leq \cdots \leq t_{o p\left(A^{\prime}-1\right)} \leq t_{o p}\left(A^{\prime}\right) .
\end{aligned}
$$

Step 3 (calculating the start operation time point set of freight operation sites). Let $t_{p(1)}^{\text {start }}$ be the start operation time point of the first freight operation site $p(1)$ in placing-in sequence $P^{\text {placing-in }}$. Obviously, we have $t_{p(1)}^{\text {start }}=t_{o, p(1)}$, where $t_{o, p(1)}$ is the travel time from railway station $o$ to the first freight operation site $p(1)$. And then, the start operation time point of the next freight operation site $p(2)$ can be written as $t_{p(2)}^{\text {start }}=t_{p(1)}^{\text {start }}+t_{p(1), p(2)}=t_{o, p(1)}+t_{p(1), p(2)}$, where $t_{p(1), p(2)}$ is the travel time from freight operation site $p(1)$ to site $p$ (2). In this way, we can have $t_{p\left(A^{\prime}\right)}^{\text {start }}=t_{p\left(A^{\prime}-1\right)}^{\text {start }}+$ $t_{p\left(A^{\prime}-1\right), p\left(A^{\prime}\right)}=t_{o, p}(1)+t_{p(1), p(2)}+\cdots+t_{p\left(A^{\prime}-1\right), p\left(A^{\prime}\right)}$. So the start time point set of freight operation sites is expressed as follows:

$$
\begin{aligned}
T^{\text {start }}= & \left\{t_{p(\delta)}^{\text {start }} \mid t_{p(1)}^{\text {start }}=t_{o, p(1)}, t_{p(\delta-1)}^{\text {start }}+t_{p(\delta-1), p(\delta)},\right. \\
& \left.\delta=2, \ldots, A^{\prime}\right\} .
\end{aligned}
$$

Step 4 (counting the operation time set of freight operation sites). We define the loading or unloading time per wagon as $\xi$ and the wagon number of the local wagon group at freight operation site $p(\delta)$ as $\omega_{p(\delta)}$. We can have

$$
U^{\text {operation }}=\left\{u(\delta) \mid u(\delta)=\xi \omega_{p(\delta)}, \delta=1, \ldots, A^{\prime}\right\} .
$$

Step 5 (calculating the completion time point set of freight operation sites). Let $t_{p(\delta)}^{\text {completion }}$ be the completion time point of loading or unloading at the freight operation site $p(\delta)$. Obviously, we have $t_{p(\delta)}^{\text {completion }}=$ $t_{p(\delta)}^{\text {start }}+u(\delta)$. So we can obtain the completion time point set of freight operation sites and be expressed as follows:

$$
\begin{aligned}
T^{\text {completion }} & =T^{\text {start }}+U^{\text {operation }}=\left\{t_{p(\delta)}^{\text {completion }} \mid t_{p(\delta)}^{\text {completion }}\right. \\
& \left.=t_{p(\delta)}^{\text {start }}+u(\delta), \delta=1, \ldots, A^{\prime}\right\} .
\end{aligned}
$$

Step 6 (generating taking-out freight operation sites set). Loaded wagons need not to be retrieved after they have finished unloading at relevant freight operation sites. But empty wagons must be retrieved after they have finished loading. So the taking-out freight operation site set is different from placing-in freight operation site set $S$. We define as $\widetilde{S}=\{1, \ldots, \widetilde{A}\}$, where $\widetilde{A}$ is the total number of taking-out freight operation sites.

Step 7 (determining taking-out freight operation site selection sequence). According to the completion time set of freight operation sites, we rank the freight operation sites for the completion time from early to later. So the set of taking-out freight operation site selection sequence $Q^{\text {taking-out }}$ can be obtained. We denote $\delta^{\prime}$ as the serial number of taking-out freight operation site. Thus, we can express $Q^{\text {taking-out }}$ as

$$
\begin{aligned}
& Q^{\text {taking-out }}=\left\{q\left(\delta^{\prime}\right) \mid q\left(\delta^{\prime}\right)=q, q \in \widetilde{S}, \delta^{\prime}=1, \ldots, \widetilde{A}\right\}, \\
& t_{q(1)}^{\text {completion }} \leq t_{q(2)}^{\text {completion }} \leq \cdots \leq t_{q(\widetilde{A}-1)}^{\text {completion }} \leq t_{q(\widetilde{A})}^{\text {completion }} .
\end{aligned}
$$

Overall, the hierarchical structure of this module is outlined in Figure 4.

4.1.2. Selecting Operation Sites Sequence Based on Maximizing Absolute Detention Time and Earliest Completion 


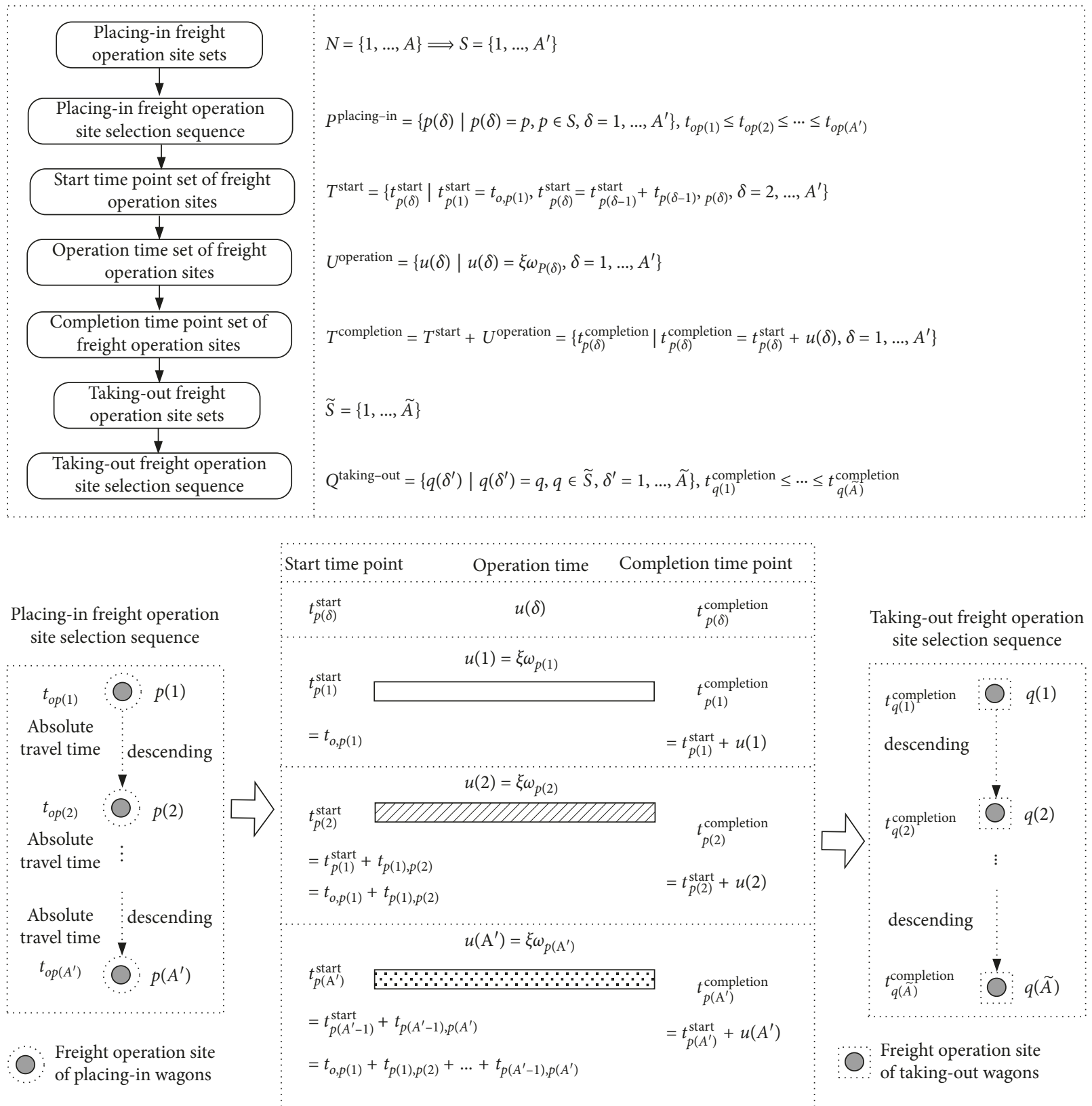

Figure 4: Hierarchical structure of the simulation module for ATT-ECT shunting strategy.

Time. Based on the hierarchical structure of ATT-ECT shunting strategy abovementioned, the procedures for shunting strategy of placing-in site sequence and taking-out site sequence based on minimizing absolute detention time and earliest completion time are given. In order to facilitate representing the problem, this shunting strategy can be abbreviated to name as ADT-ECT. Because the difference between the new ADT-ECT strategy and above ATT-ECT strategy is only reflected in the second step, and the method of generating placing-in freight operation site selection sequence is provided as follows.

We define the absolute detention time as $\tilde{t}_{o j}$. It is the sum of the travel time $t_{o j}$ from railway station $o$ to freight operation site $j$ and operation time $u(j)=\xi \omega_{j}$, i.e., $\tilde{t}_{o j}=t_{o j}+u(j)$. Here, $\xi$ denotes the loading or unloading time per wagon and $\omega_{j}$ represents the wagon number of local wagon group at freight operation site $j$. And then, we count the absolutely detention time $\widetilde{t}_{o j}$ and rank the freight operation sites in the order of absolutely detention time from high to low. So the placing-in freight operation site selection sequence $P^{\text {placing-in }}$ can be obtained. We denote $\delta$ as the serial number of placing-in freight operation site. Thus, $P^{\text {placing-in }}$ can be expressed as follows:

$$
\begin{aligned}
& P^{\text {placing-in }}=\left\{p(\delta) \mid p(\delta)=p, p \in S, \delta=1, \ldots, A^{\prime}\right\} \\
& \tilde{t}_{o p(1)} \geq \widetilde{t}_{o p(2)} \geq \cdots \geq \widetilde{t}_{o p\left(A^{\prime}-1\right)} \geq \widetilde{t}_{o p\left(A^{\prime}\right)} .
\end{aligned}
$$

Overall, the procedure of placing-in freight operation site selection sequence for ADT-ECT shunting strategy is outlined in Figure 5. 


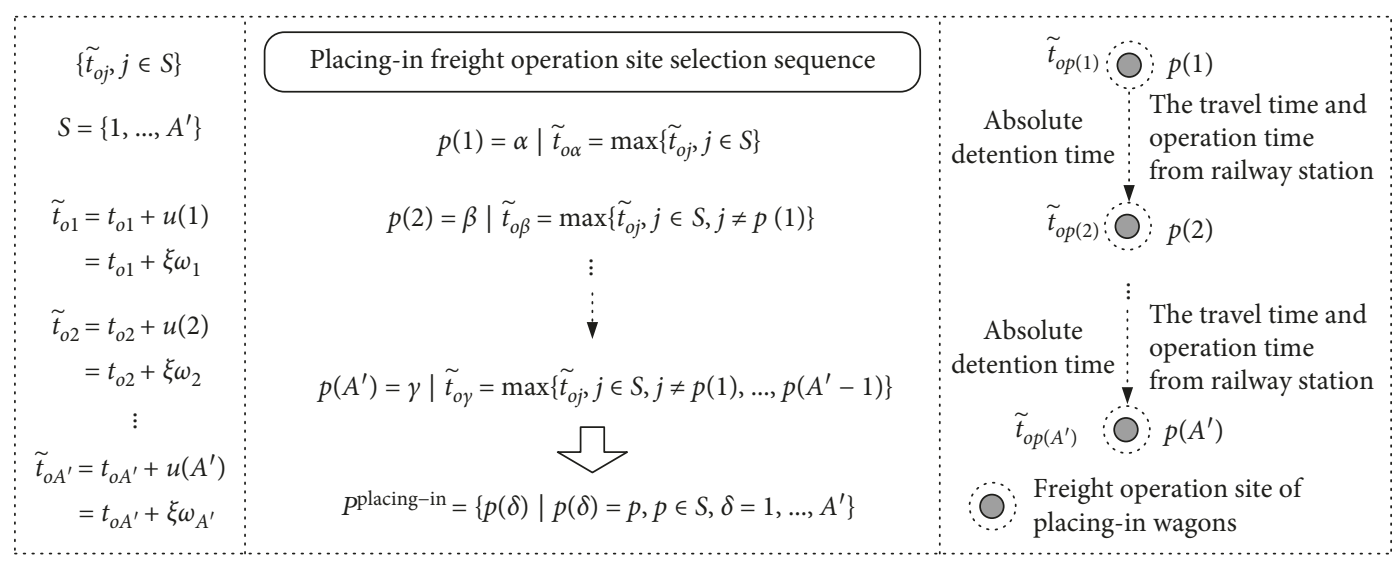

Figure 5: Procedure of placing-in freight operation site selection sequence for ADT-ECT shunting strategy.

4.1.3. Selecting Operation Sites Sequence Based on Minimizing Relative Travel Time and Earliest Completion Time. Shunting strategy of placing-in site sequence and taking-out site sequence based on minimizing relative travel time and earliest completion time is abbreviated as RTT-ECT in order to facilitate representing the problem. Except for the method of the generating placing-in freight operation site selection sequence, the RTT-ECT strategy is exactly the same as the ATT-ECT strategy. So we develop only this method as follows.

We count the absolute travel time $t_{o j}$ from railway station $o$ to any freight operation site $j \in S$. We select the shortest absolute travel time $t_{o \alpha}$ and make the relevant freight operation site $\alpha$ as the first freight operation site $p(1)$ in placing-in sequence $P^{\text {placing-in }}$. And then, we let the freight operation site.

$\alpha$ is the current freight operation site. From the destination freight operation site set $S$, we find the nearest site $\beta$ from current site $\alpha$ and arrange it as the next current site $p(2)$. In this way, the placing-in freight operation site selection sequence $P^{\text {placing-in }}$ can be obtained. So we have

$$
P^{\text {placing-in }}=\left\{p(\delta) \mid p(\delta)=p, p \in S, \delta=1, \ldots, A^{\prime}\right\},
$$

where

$$
\begin{aligned}
p(1)= & \alpha \mid t_{o \alpha}=\min \left\{t_{o j}, j \in S\right\}, \\
p(2)= & \beta \mid t_{p(1) \beta}=\min \left\{t_{\alpha j}, j \in S, j \neq p(1)\right\}, \ldots, \\
p\left(A^{\prime}\right)= & \gamma \mid t_{p\left(A^{\prime}-1\right) \gamma}=\min \left\{t_{p\left(A^{\prime}-1\right) j}, j \in S, j \neq p(1), \ldots,\right. \\
& \left.p\left(A^{\prime}-1\right)\right\} .
\end{aligned}
$$

Overall, the procedure of placing-in freight operation site selection sequence for the RTT-ECT shunting strategy is outlined in Figure 6.

4.1.4. Selecting Operation Sites Sequence Based on Maximizing Relative Detention Time and Earliest Completion Time. Here, this shunting strategy is abbreviated as RDTECT. Similarly, we develop the method of the generating placing-in freight operation site selection sequence which is the only difference from RTT-ECT strategy mentioned above.

We count the absolute detention time $\tilde{t}_{o j}$ from railway station $o$ to any freight operation site $j \in S$. The freight operation site $\alpha$ which is the longest absolute detention time from railway station $o$ is arranged as the first freight operation site $p(1)$ in placing-in sequence $P^{\text {placing-in }}$. And then, we find the site $\beta$ which is the longest detention time $\tilde{t}_{\alpha \beta}$ from current site $\alpha$ and arrange it as the next current site $p(2)$. In this way, the placing-in freight operation site selection sequence $P^{\text {placing-in }}$ can be obtained and expressed as follows:

$$
P^{\text {placing-in }}=\left\{p(\delta) \mid p(\delta)=p, p \in S, \delta=1, \ldots, A^{\prime}\right\},
$$

where

$$
\begin{aligned}
p(1)= & \alpha \mid \tilde{t}_{o \alpha}=\max \left\{\widetilde{t}_{o j}, j \in S\right\}, \\
p(2)= & \beta \mid \tilde{t}_{p(1) \beta}=\max \left\{\widetilde{t}_{\alpha j}, j \in S, j \neq p(1)\right\}, \ldots, \\
p\left(A^{\prime}\right)= & \gamma \mid \widetilde{t}_{p\left(A^{\prime}-1\right) \gamma}=\max \left\{\widetilde{t}_{p\left(A^{\prime}-1\right) j}, j \in S, j \neq p(1), \ldots,\right. \\
& \left.p\left(A^{\prime}-1\right)\right\} .
\end{aligned}
$$

Overall, the procedure of placing-in freight operation site selection sequence for the RTT-ECT shunting strategy is outlined in Figure 7.

4.1.5. Selecting Operation Sites Sequence Based on Nearest Location and Completion Time. The abovementioned four shunting strategies, i.e., ATT-ECT, ADT-ECT, RTT-ECT, and RDT-ECT, all generate taking-out freight operation site sequence using earliest completion time. Instead of this regulation, we will provide another four different shunting strategies which generate taking-out freight operation site sequence with nearest location and completion time. Thus, we will obtain four new shunting strategies, i.e., placing-in and taking-out site sequence based on minimizing absolute travel time and nearest location and completion time (ATTNL\&CT), placing-in and taking-out site sequence based on maximizing absolute detention time and nearest location 


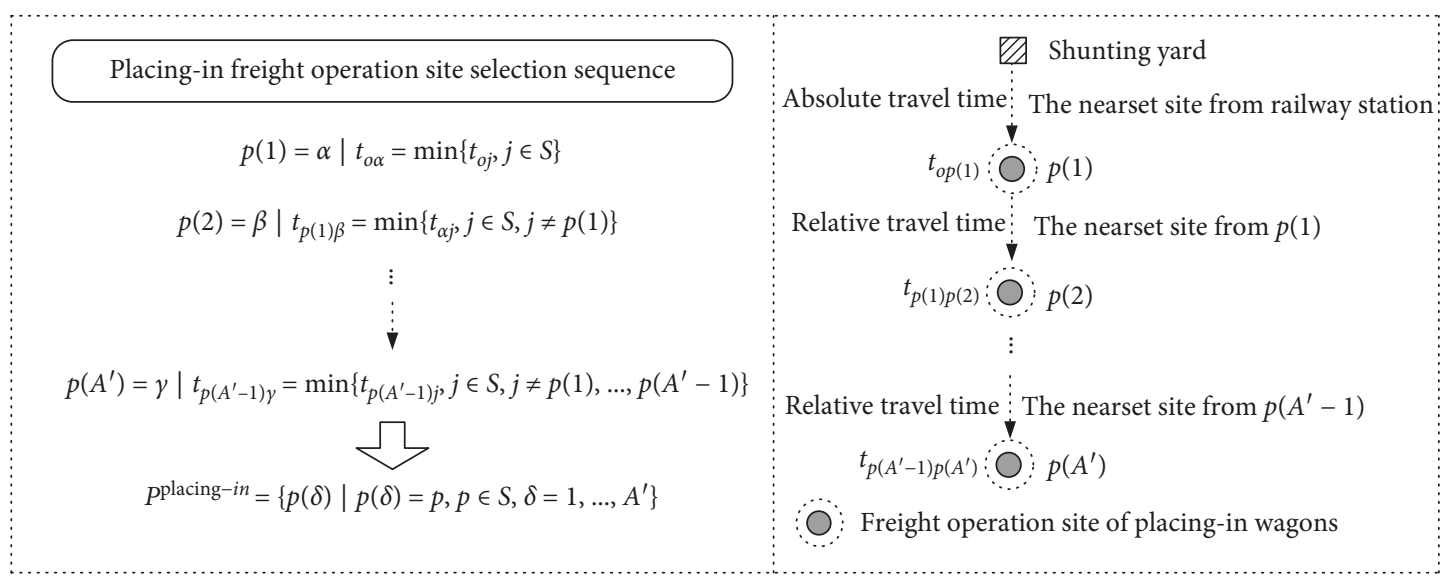

FIGURE 6: Procedure of placing-in freight operation site selection sequence for the RTT-ECT shunting strategy.

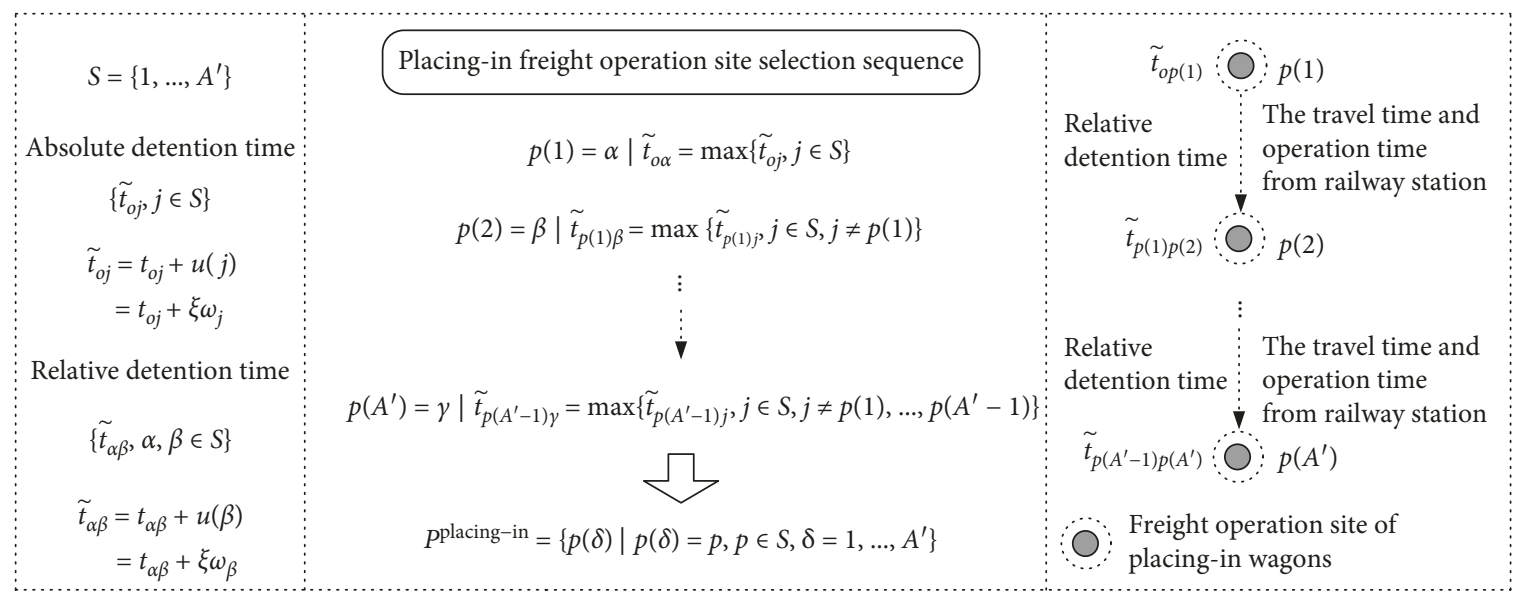

FIGURE 7: Procedure of placing-in freight operation site selection sequence for the RTT-ECT shunting strategy.

and completion time (ADT-NL\&CT), placing-in and takingout site sequence based on minimizing relative travel time and nearest location and completion time (RTT-NL\&CT), and placing-in and taking-out site sequence based on maximizing relative detention time and nearest location and completion time (RDT-NL\&CT). To avoid repeated expression, we provide only the procedure of generating taking-out freight operation site selection sequence.

From the last placing-in freight operation site $p\left(A^{\prime}\right)$, we begin to generate taking-out sequence $Q^{\text {taking-out }}$ with reverse tracing. According to the completion time point, the available taking-out site set $M$ is given by

$$
\begin{aligned}
M & =\left\{m \mid m=j, t_{p\left(A^{\prime}\right)}^{\text {complion }}+t_{p\left(A^{\prime}\right) j} \geq t_{j}^{\text {completion }}, j \in \widetilde{S}\right\}, \\
\widetilde{S} & =\{1, \ldots, \widetilde{A}\} .
\end{aligned}
$$

According to the nearest site regulation, the first takingout site can be obtained using $q(1)=\min \left\{m \mid t_{p\left(A^{\prime}\right) m}\right.$, $m \in M\}$. From $q(1)$, we begin to find the second taking-out site $q(2)$. The new available taking-out site set $M$ can be written as

$$
\begin{aligned}
M= & \left\{m \mid m=j, t_{p\left(A^{\prime}\right)}^{\text {completion }}+t_{p\left(A^{\prime}\right) q(1)}+t_{q(1) j} \geq t_{j}^{\text {completion }},\right. \\
& j \in \widetilde{S}, j \neq q(1)\} \quad \widetilde{S}=\{1, \ldots, \widetilde{A}\} .
\end{aligned}
$$

And then, the second taking-out site can be given using $q(2)=\min \left\{m \mid t_{q(1) m}, m \in M\right\}$. And so on, the last takingout site can be obtained by

$$
\begin{aligned}
& M=\left\{m \mid m=j, t_{p\left(A^{\prime}\right)}^{\text {completion }}+t_{p\left(A^{\prime}\right) q(1)}+t_{q(1) q(2)} \cdots\right.
\end{aligned}
$$

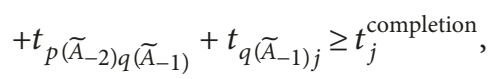

$$
\begin{aligned}
& j \in \widetilde{S}, j \neq q(1), \ldots, q(\widetilde{A}-1)\}, \\
& q(\widetilde{A})=\min \left\{m \mid t_{q(\tilde{A}-1) m}, m \in M\right\}, \quad \widetilde{S}=\{1, \ldots, \widetilde{A}\} .
\end{aligned}
$$

Overall, the set of taking-out freight operation site selection sequence $Q^{\text {taking-out }}$ can be obtained according to the nearest location and completion time. The hierarchical structure of this module is outlined in Figure 8. 


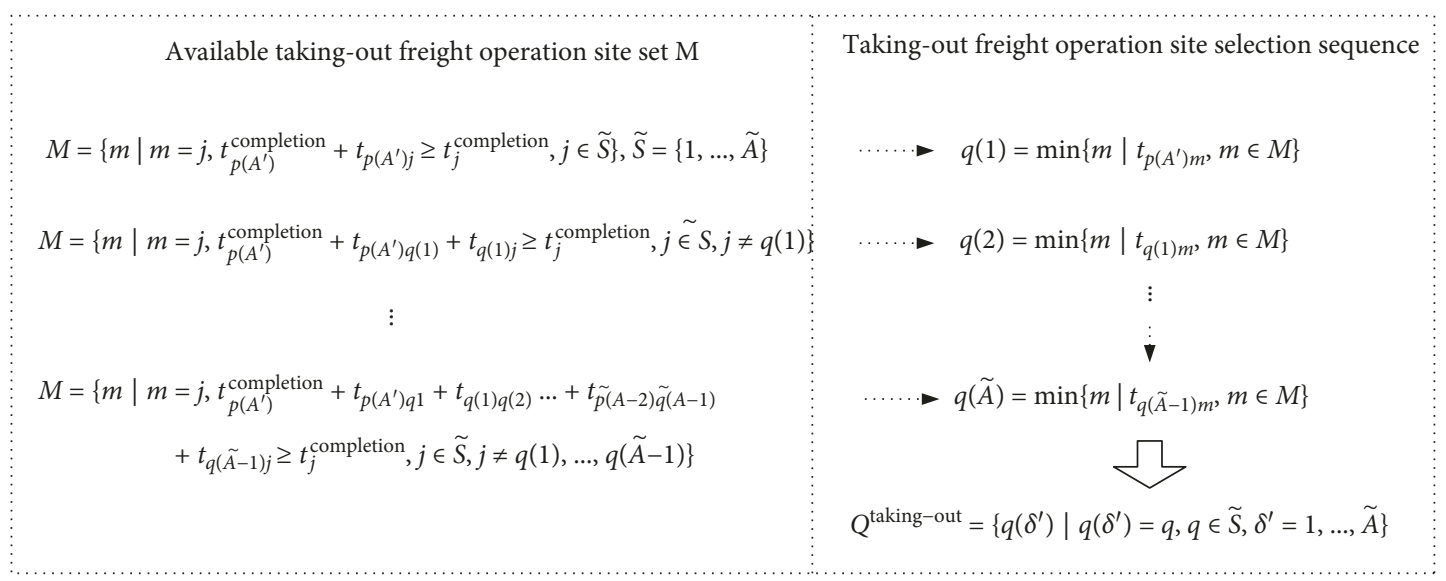

FIGURE 8: Procedure of taking-out freight operation site selection sequence with NL\&CT.

4.2. Simulating PTWS-SSMS. Under scenarios of PTWSSSMS, there are some double operation wagons in the local wagons. These double local wagons are usually loaded wagons. They will be moved to another freight site for loading after the double local wagon group is detached from shunting locomotive and has finished the unloading at relevant freight operation site. So the span-shift-moving shunting generates. Except for span-shift-moving shunting which does not appear in PTWS-SO, the hierarchical structure of shunting strategies for PTWS-SSMS is exactly the same as that of PTWS-SO. To avoid repeated expression, we provide only the procedure of span-shift-moving shunting. Here, we present two different span-shift-moving strategies, i.e., span-shift-moving shunting based on earliest completion time and span-shift-moving shunting based on nearest location and completion time.

4.2.1. Span-Shift-Moving Shunting Based on Earliest Completion Time. Span-shift-moving shunting based on earliest completion time is abbreviated as SSM-ECT. We develop the procedure of the strategy as follows.

Step 1 (generating shift-out freight operation sites set). We take the unloading freight operation sites of double operation wagons as shift-out freight operation sites. So we denote the shift-out freight operation sites set as $R=\{1, \ldots, B\}$, where $B$ is the total number of shift-out freight operation sites.

Step 2 (determining shift-moving sequence). According to the completion time point of shift-out freight operation sites, we rank the shift-out freight operation sites for the completion time point from early to later. From the last placing-in freight operation site $p\left(A^{\prime}\right)$, we take the priority on the shift-out sites with earlier operation completion time point until the last shift-out site. So the shift-moving sequence $Z^{\text {shift-moving }}$ can be obtained. Thus, we can express $Z^{\text {shift-moving }}$ as

$$
\begin{aligned}
Z^{\text {shift- moving }=} & \left\{p\left(A^{\prime}\right), z(\theta)-\tilde{z}(\theta), \tilde{z}(\theta)-z(\theta+1)\right. \\
& \mid \theta=1, \ldots, B-1\}, \\
t_{z(1)}^{\text {completion }} \leq & t_{z(2)}^{\text {completion }} \leq \cdots \leq t_{z(B-1)}^{\text {completion }} \leq t_{z(B)}^{\text {completion }},
\end{aligned}
$$

where $z(\theta)$ denotes shift-out site and $\widetilde{z}(\theta)$ denotes the shiftinto site of shift-out site $z(\theta)$.

Overall, the procedure of SSM-ECT for PTWS-SSMS is outlined in Figure 9.

4.2.2. Span-Shift-Moving Shunting Based on Nearest Location and Completion Time. Span-shift-moving shunting based on nearest location and completion time is abbreviated as SSM-NL\&CT. The procedure of the strategy is developed as follows.

Similarly, we define the shift-out freight operation sites set as $R=\{1, \ldots, B\}$, where $B$ is the total number of shift-out freight operation sites. From the last placing-in freight operation site $p\left(A^{\prime}\right)$, we begin to generate shift-moving sequence $Z^{\text {shift-moving }}$.

According to the completion time point, the available shift-out site set $U$ is given by the following equation:

$$
\begin{aligned}
U= & \left\{u \mid u=j, t_{p\left(A^{\prime}\right)}^{\text {completion }}+t_{p\left(A^{\prime}\right) j} \geq t_{j}^{\text {completion }}, j \in R\right\}, \\
& R=\{1, \ldots, B\} .
\end{aligned}
$$

According to the nearest site regulation, the first shiftout site can be obtained using $z(1)=\min \left\{u \mid t_{p\left(A^{\prime}\right) u}, u \in U\right\}$. And then, the double operation wagons are moved to the shift-into freight operation site $\widetilde{z}(1)$ from $z(1)$. From the shift-into freight operation site $\widetilde{z}(1)$, we begin to find the second shift-out site $z$ (2). The new available shift-out site set $U$ can be written as

$$
\begin{aligned}
U= & \left\{u \mid u=j, t_{p\left(A^{\prime}\right)}^{\text {completion }}+t_{p\left(A^{\prime}\right) z(1)}+t_{z(1) \widetilde{z}(1)}\right. \\
& \left.+t_{\widetilde{z}(1) j} \geq t_{j}^{\text {completion }}, j \in R, j \neq z(1)\right\}, \quad R=\{1, \ldots, B\} .
\end{aligned}
$$

And then, the second shift-out site can be given using $z(2)=\min \left\{u \mid t_{\widetilde{z}(1) u}, u \in U\right\}$. The double operation wagons are moved to the shift-into freight operation site $\widetilde{z}(2)$ from $z$ (2). And so on, the last shift-out site can be obtained by the following equation: 


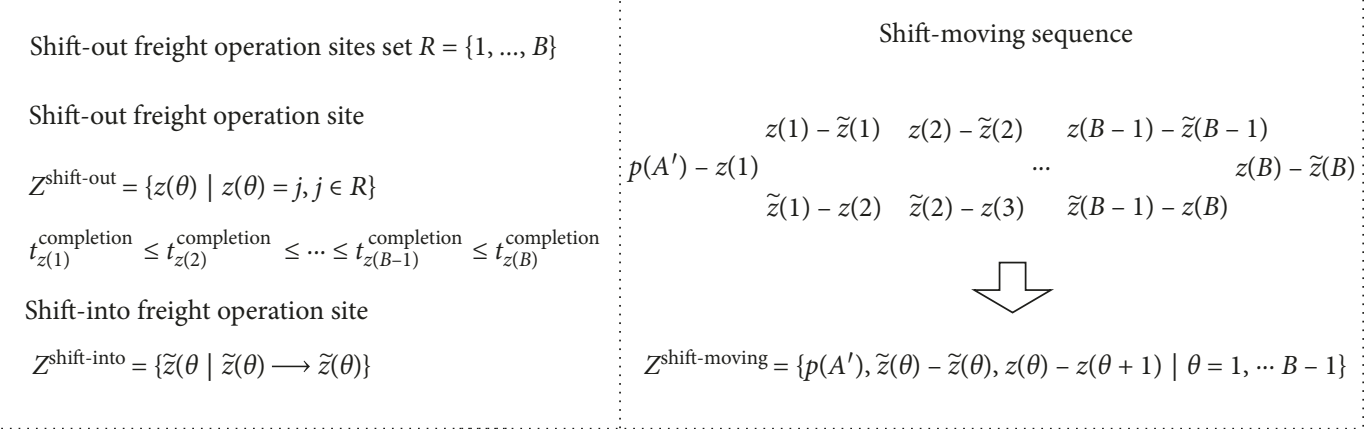

FIgure 9: Procedure of SSM-ECT for PTWS-SSMS.

$$
\begin{aligned}
U= & \left\{u \mid u=j, t_{p\left(A^{\prime}\right)}^{\text {completion }}+t_{p\left(A^{\prime}\right) z(1)}+t_{z(1) \widetilde{z}(1)}\right. \\
& +t_{\widetilde{z}(1) z(2)} \cdots+t_{z(B-1)} \widetilde{z}_{(B-1)}+t_{\widetilde{z}(B-1) j} \geq t_{j}^{\text {completion }}, \\
& j \in R, j \neq z(1), \ldots, z(B-1)\}, \\
z(B)= & \min \left\{u \mid t_{\widetilde{z}(B-1) u}, u \in U\right\}, \quad R=\{1, \ldots, B\} .
\end{aligned}
$$

So the shift-moving sequence $Z^{\text {shift-moving }}$ can be obtained. Thus, we can express $Z^{\text {shift-moving }}$ as

$$
\begin{aligned}
Z^{\text {shift-moving }}= & \left\{p\left(A^{\prime}\right), z(\theta)-\tilde{z}(\theta), \widetilde{z}(\theta)-z(\theta+1)\right. \\
& \mid \theta=1, \ldots, B-1\},
\end{aligned}
$$

where $z(\theta)$ denotes shift-out site and $\widetilde{z}(\theta)$ denotes the shiftinto site of shift-out site $z(\theta)$.

Overall, the procedure of SSM-NL\&CT for PTWS-SSMS is outlined in Figure 10.

\section{Simulation Platform Based on Arena}

In this section, we develop simulation platform of PTWSBSFON with the Arena simulation software. Our Arena simulation platform is a collection of railway station submodel, freight operation sites submodel under PTWSSO, and freight operation sites submodel under PTWSSSMS. The purpose of setting up simulation platform is to evaluate the performance of different shunting strategies under various scenarios. The logic of the PTWS simulation platform is demonstrated in Figure 11. All Arena modules used in the simulation model are listed in Table 1 .

5.1. Main Submodel for Railway Station. This section gives a short overview of the main simulation submodel for railway station employed for the numerical test conducted in this work. We identify the need to set up three main components: arrival yard component, shunting yard component, and departure yard component. The flowchart of the railway stations submodel based on the Arena simulation platform is shown as Figure 12.
5.1.1. Arrival Yard Component. Wagons' arrival is the first component of the simulation platform. We use Create module to generate wagon group entities. The time interval of wagon group entities is a random variable obeying exponential distribution. The daily arrival numbers of inbound trains obey uniform distribution. Here, we set up some input parameters denoting above random distribution function by Create module. Assign module allocates an attribution to each incoming wagon group entity. The assigned attribution represents the destination freight operation sites of wagon groups. Each destination corresponds to an attribution value. For example, if the destination of one wagon group is freight operation site 1 , we have destination attribute be 1 .

5.1.2. Shunting Yard Component. After the local wagons reach arrival yard, they are decoupled from inbound trains and accumulated at the shunting yard. Batch module is used to accumulate all local wagons. Once the local wagons entities have accumulated to a specific size, Request module will call a shunting locomotive from engine depot. After the locomotive arriving at the shunting yard, all local wagons are attached. And then, all wagon groups are dispatched to the corresponding destination according to placing-in freight operation sites selection sequence. Pickstation module is adopted for wagons entities selecting their destination operation sites. We use the expression option of this module to give a formulation for each station. The setting parameters that are used to choose first placing-in freight operation site under four placing-in wagons strategies are collected into Pickstation module.

5.1.3. Departure Yard Component. After all local wagons are pulled back and retrieved to the shunting yard, they are detached from the locomotive by Separate module. Then, these detached local wagons are sorted into new outbound trains and leave system from the departure yard by Dispose module. All wagons entities get into Dispose module as the ending of simulation.

5.2. Main Submodel for Freight Operation Sites under PTWSSO. This section gives a short overview of the main simulation submodel for freight operation sites under PTWS-SO employed for the numerical test conducted in this work. We 


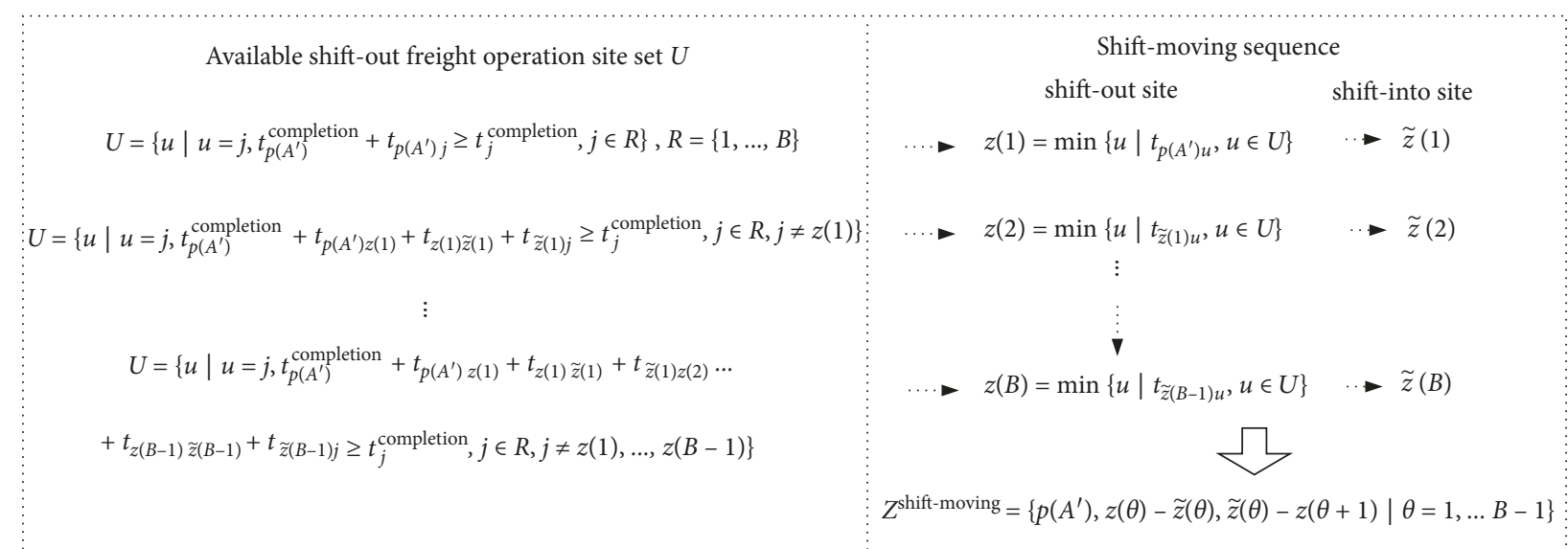

FIGURE 10: Procedure of SSM-NL\&CT for PTWS-SSMS.

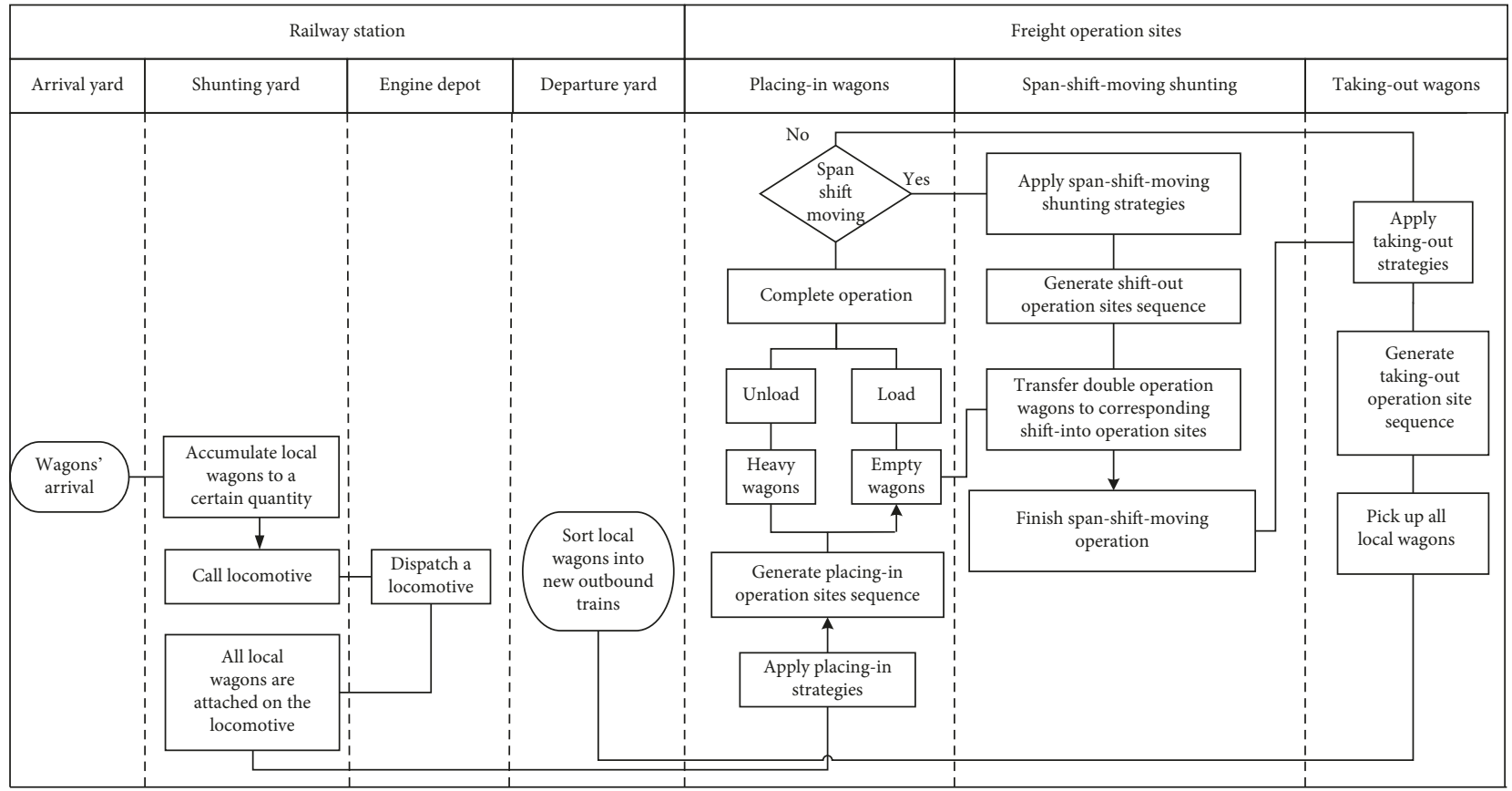

FIgURE 11: Logic of the PTWS simulation platform.

identify three main components for the simulation submodel: operation component, placing-in wagons component, and taking-out wagons component. The flowchart of freight operation sites submodel under PTWS-SO based on the Arena simulation platform is shown as Figure 13.

5.2.1. Operation Component. While the locomotive arrives at one freight operation site under PTWS-SO, it firstly enters into Decide module. Decide module provides a decisionmaking process to identify placing-in wagons or taking-out wagons. If placing-in wagons is finished, the locomotive picks up local wagons. Otherwise, it enters into Search and Remove modules. Then, Search and Remove modules select a specific wagon group by search condition. Wagon group meeting search condition is detached from shunting locomotive and then sent to right place for handling. Empty wagon groups are loaded, and heavy wagons groups are unloaded.

Assign module is used to define start operation time point of freight operation site $i$ as Start Time $i$. Let the value of variable Start Time $i$ be current simulation time in Arena software. The current simulation time is output to a file through ReadWrite module. And then, we use furtherly Assign module to denote Placing-in Helper $i$ as auxiliary variables. The auxiliary variables are introduced to avoid duplicate accessing the same freight operation site. Process module is adopted to denote local wagons handling in their destination freight operation sites. The wagons entities cannot leave Process module until operation has been completed. The operation time of the wagon group is a random variable obeying uniform distribution. The "Delay Type" option in Process module is set as uniform to denote above uniform distribution. We use Assign module to define 
TABLe 1: List of all Arena modules used in the simulation model.

\begin{tabular}{lccc}
\hline Module & Name & Module & Name \\
\hline Create & Wagon group $i$ & Pickstation & Choose the first placing-in operation site \\
Assign & Destination is operation site $i$ & Pickstation & Travel to next placing-in operation site \\
Assign & Start time $i$ & Pravel to first taking-out operation site \\
Assign & Completion time $i$ & Pickstation & Travel to next taking-out operation site \\
Assign & Completion helper $i$ & Pickstation & Travel to the first shift-out operation site \\
Assign & Taking-out time $i$ & Pickstation & Travel to next shift-out operation site \\
Assign & Taking-out helper $i$ & Decide & Finish placing-in wagons \\
Assign & Shift-out time $i$ & Wecide & Wait \\
Station & Span shift-moving helper $i$ & Decide & Finish taking-out wagons \\
Station & Operation site $i$ & Decide & Last placing-in operation site \\
Station & Shift-out operation site & Decide & Span-shift-moving shunting operation is finished \\
Station & Shift-into operation site & Pickup & Pickup wagon group \\
Station & Shunting yard & Pickup & Pickup double operation wagons \\
Request & Call the locomotive & Route & Return to shunting yard \\
Request & Locomotive & Route & Travel to corresponding shift-into operation site \\
Batch & Accumulate local wagons & ReadWrite & Output start time $i$ \\
Separate & Detach all local wagons & ReadWrite & Output completion time $i$ \\
Dispose & Departure yard & ReadWrite & Output taking-out time $i$ \\
Search and remove & Select specific wagon group & ReadWrite & Output shift-out time $i$ \\
Process & Operation & Hold & Hold wagon group in current operation site \\
\hline
\end{tabular}
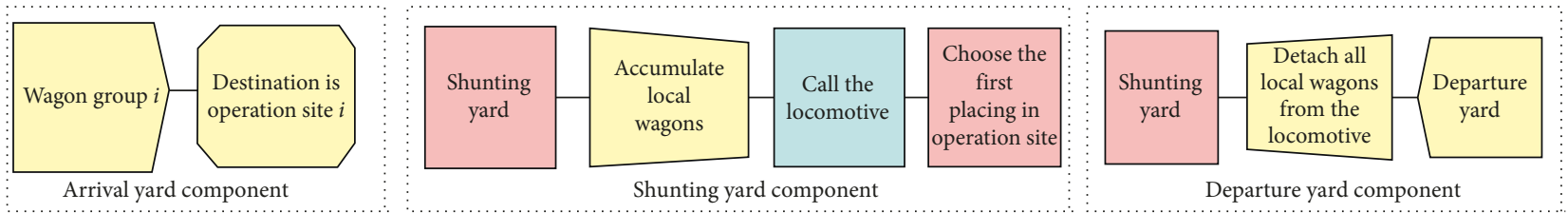

FIGURE 12: Flowchart of submodel for a railway station based on the Arena simulation platform.

completion time point of freight operation site $i$ as Completion Time $i$. Let the value of variable Completion Time $i$ be current simulation time in Arena software. The current simulation time is output to a file through ReadWrite module. And then, we use furtherly Assign module to denote Completion Helper $i$ as auxiliary variables. The auxiliary variables are introduced to identify whether the handling is finished or not in freight operation site $i$. After the handling is finished, local wagons wait in Hold module until the shunting locomotive picks them up.

5.2.2. Placing-In Wagons Component. Local wagon groups are separated into two parts by Search and Remove modules. One part is the wagon group whose destination is the current freight site. The rest enters into another Decide module to identify if current freight operation site is the last of placingin operation site selection sequence. If it does, we adopt another Decide module named as Wait to make a decision whether or not taking-out wagons begins. If any handling at all operation sites has not been completed at this time point, the locomotive should be held in Hold module until appearing the freight operation site where the handling has been finished. Else, the locomotive travels to the first of taking-out freight operation sites selection sequence by Pickstation module immediately. The setting parameters that are used to develop Pickstation module for travelling to the first taking-out freight operation site under two taking- out strategies are collected into Pickstation module. If current operation site is not the last of placing-in operation sites sequence, the locomotive continues travelling to the next operation site of placing-in freight operation sites selection sequence by Pickstation module. The setting parameters that are used to develop Pickstation module for travelling to next freight operation site in the placing-in process under four strategies are collected into Pickstation module.

5.2.3. Taking-Out Wagons Component. When the locomotive reaches one destination freight operation site to pick up wagons, Assign module is used to define taking-out time point of freight operation site $i$ as taking-out time $i$. Let the value of variable taking-out time $i$ be current simulation time in Arena software. The current simulation time is output to a file through ReadWrite module. Pickup module is applied to attach the wagon group in current freight operation site on the locomotive. And then, we use furtherly Assign module to denote taking-out helper $i$ as auxiliary variables. The auxiliary variables are introduced to avoid duplicate accessing the same freight operation site in the taking-out wagons process. After that, the locomotive enters into Decide module. Decide module provides a decision-making process to identify whether taking-out wagons is finished or not. If the current operation site is the end site in taking-out freight operation sites selection sequence, the locomotive must 


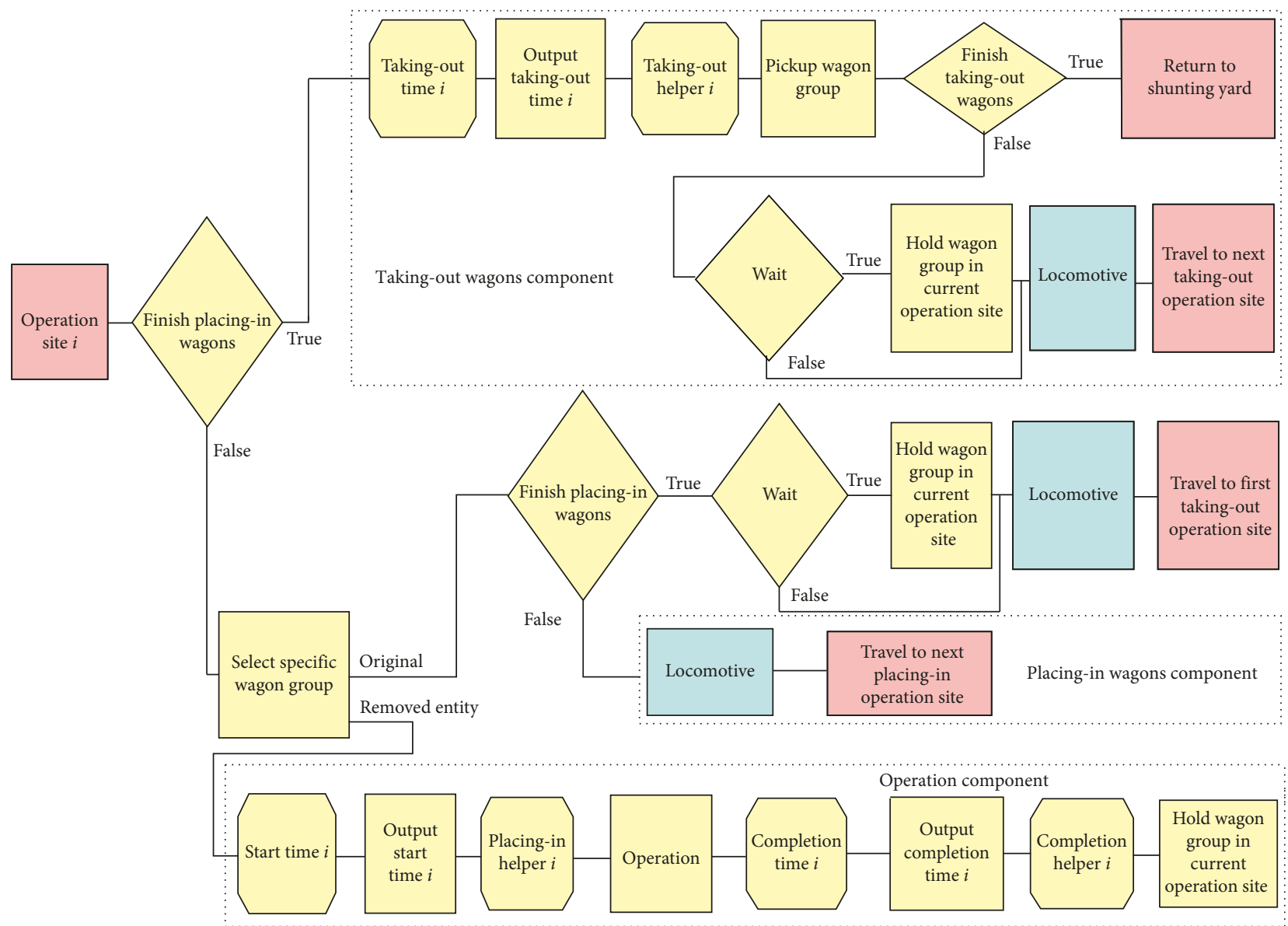

FIGURE 13: Flowchart of submodel for freight operation sites under PTWS-SO based on the Arena simulation platform.

return to the shunting yard by Route module. Otherwise, the locomotive enters into the Decide module mentioned in above section and travels to next taking-out operation site by Pickstation module. The setting parameters that are used to develop Pickstation module for travelling to next taking-out freight operation site under two strategies are collected into Pickstation module.

5.3. Main Submodel for Freight Operation Sites under PTWSSSMS. This section gives a short overview of the main simulation submodel for freight operation sites under PTWS-SSMS employed for the numerical test conducted in this work. We identify the need to model three types of freight operation sites: operation freight sites without spanshift-moving shunting, shift-out operation sites, and shiftinto operation sites.

5.3.1. Freight Operation Sites without Span-Shift-Moving Shunting. Here, we set up four components for this type freight operation site: placing-in component, operation component, start span-shift-moving shunting component, and taking-out component. The placing-in component, operation component, and taking-out component are the same as those in freight operation sites under PTWS-SO. To avoid repeated expression, we only introduce start span- shift-moving shunting component. Span-shift-moving shunting is carried out between placing-in wagons and taking-out wagons. After placing-in wagons is finished, the operation of span-shift-moving shunting starts by Pickstation module. The setting parameters that are used to develop Pickstation module for travelling to the first shiftout operation site under two strategies are collected into Pickstation module. The flowchart of the submodel for freight operation sites without span-shift-moving shunting based on the Arena simulation platform is shown as Figure 14 .

5.3.2. Shift-Out Freight Operation Sites. Here, we model three components for this type freight operation site: placing-in component, operation component, and shift-out component. The placing-in component and operation component are the same as those in freight operation sites under PTWS-SO. We only introduce shift-out component. When the locomotive arrives at one shift-out freight operation site, Assign module is used to define shift-out time point of freight operation site $i$ as Shift-out Time $i$. Let the value of variable Shift-out Time $i$ be current simulation time in Arena software. The current simulation time is output to a file through ReadWrite module. Pickup module is used to pick up the double operation wagon group in current freight 


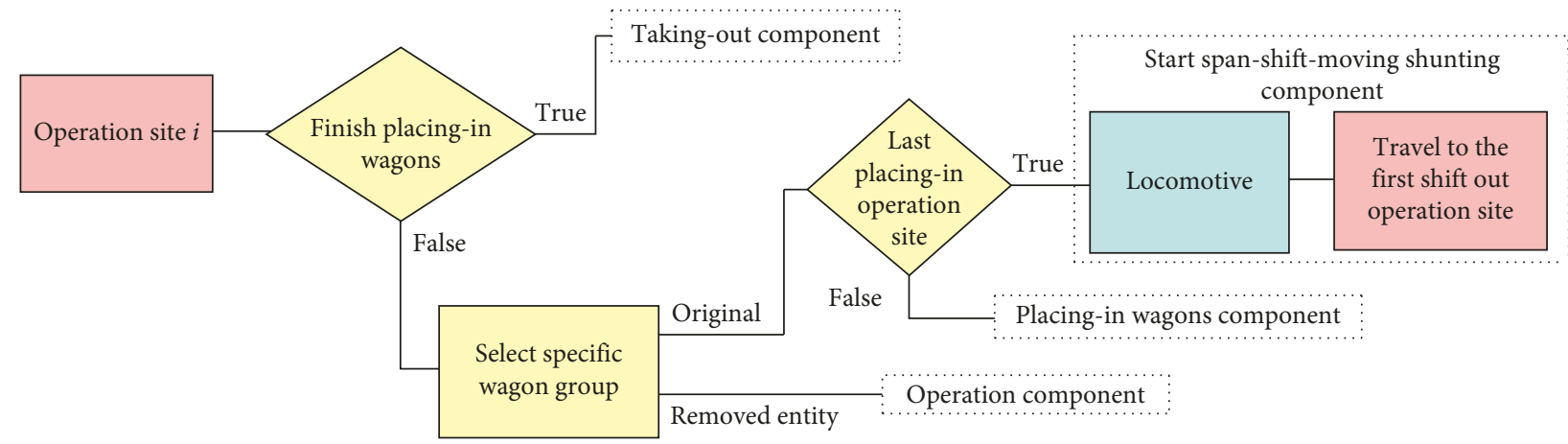

FIGURE 14: Flowchart of submodel for freight operation sites without span-shift-moving shunting based on the Arena simulation platform.

site. Then, Assign module is applied to define Span-shiftmoving Helper $i$ as auxiliary variables. The auxiliary variables are introduced to avoid duplicate accessing the same shift-out freight operation site in the span-shift-moving shunting process. Then, the locomotive travels to corresponding shift-into freight operation site by Route module. The flowchart of the submodel for shift-out freight operation sites based on the Arena simulation platform is shown as Figure 15.

5.3.3. Shift-Into Freight Operation Sites. We provide four components for this type freight operation site: placing-in component, operation component, span-shift-moving shunting component, and taking-out component. The placing-in component, operation component, and takingout component are the same as those in freight operation sites under PTWS-SO. We only introduce span-shiftmoving shunting component. After the locomotive arrives at corresponding shift-into freight operation site, it enters into Decide module to identify whether all span-shift-moving shunting are finished or not. If span-shift-moving shunting is completed, taking-out wagons is carried out. Otherwise, the locomotive travels to next shift-out freight operation site of shift-moving sequence by Pickstation module. The setting parameters that are used to develop Pickstation module for travelling to next shift-out freight operation site under two span-shift-moving shunting strategies are collected into Pickstation module. The flowchart of the submodel for shiftinto freight operation sites based on Arena simulation platform is shown as Figure 16.

\section{Case Study}

In this section, we present two PTWS-BSFON types, and the data sets are actually chosen to be used in the experiments designed to answer each of the research questions. Two PTWS-BSFON types, i.e., PTWS-SO and PTWS-SSMS, are, respectively, chosen for the tests which deal with the different shunting strategies on PTWS-BSFON, optimization of PTWS-BSFON, and monitoring of PTWS-BSFON.

6.1. Scenario Settings of the Experiments. To design the experiments scheme, the scenarios settings need to be firstly set up. We generated the dataset based on the Zhengzhou railway terminal of China. However, the data have some inconsistencies caused by the time interval of the inbound trains, the total number of local wagons, wagons number of each wagons group being dispatched to various freight operation sites, loading or unloading time of wagons group being dispatched to various freight operation sites, and so on. Therefore, the data have to be cleaned. Because the occasional result of single experiment cannot be avoided, the experiment is repeatedly tested to 10 times under the condition of the same initial conditions and termination criteria.

Here, we designed the experiments according to the throughput of the Zhengzhou railway terminal. The daily arrival numbers of inbound trains are the random variables between 30 and 40 . The interval times of the inbound trains obey exponential distribution, and its expected value is the certain range from 30 minutes to 50 minutes. We give shunting locomotive velocities of 30 kilometers per hours. The loading or unloading time of each wagon is allowed the certain range from 20 minutes to 30 minutes.

To evaluate the performance of the simulation platform proposed, we generate randomly two sets of problem instances with certain number of freight operation sites. Two sets of experiments are oriented to test the performances of simulation module based on different shunting strategies using the ARENA platform. In the first set of contrast experiment, the number of the sites is 8 in the branch-shaped freight operation network (BSFON) and the experiment is designed to test PTWS-SO. In the second set of contrast experiment, the number of the sites is the same and the experiment is used to test PTWS-SSMS. In two experiments, the distance among freight operation sites is designed to be approximately between $2 \mathrm{~km}$ and $10 \mathrm{~km}$, refer to the layout of the Zhengzhou railway terminal.

The experiments are run by the simulation platform. The objectives of this experiment are twofold. The first objective is to give some special attention to testing the performances of different shunting strategies on PTWS-BSFON, which measure the total detention time and waiting time caused by different shunting strategies. The second objective is to provide the optimal shunting strategy for enhancing the performance of PTWS-BSFON.

6.2. Testing Performance of PTWS-SO. Modeling by the method of the simulation platform can consider all kinds of 


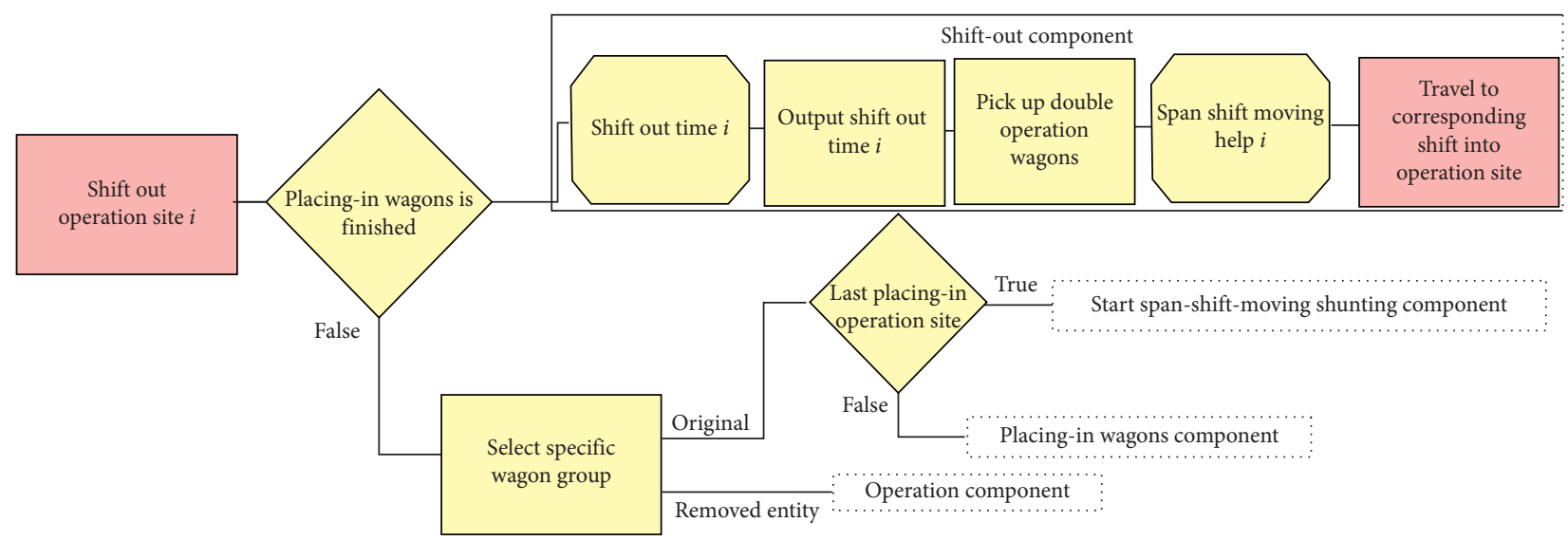

FIGURE 15: Flowchart of submodel for shift-out freight operation sites based on the Arena simulation platform.

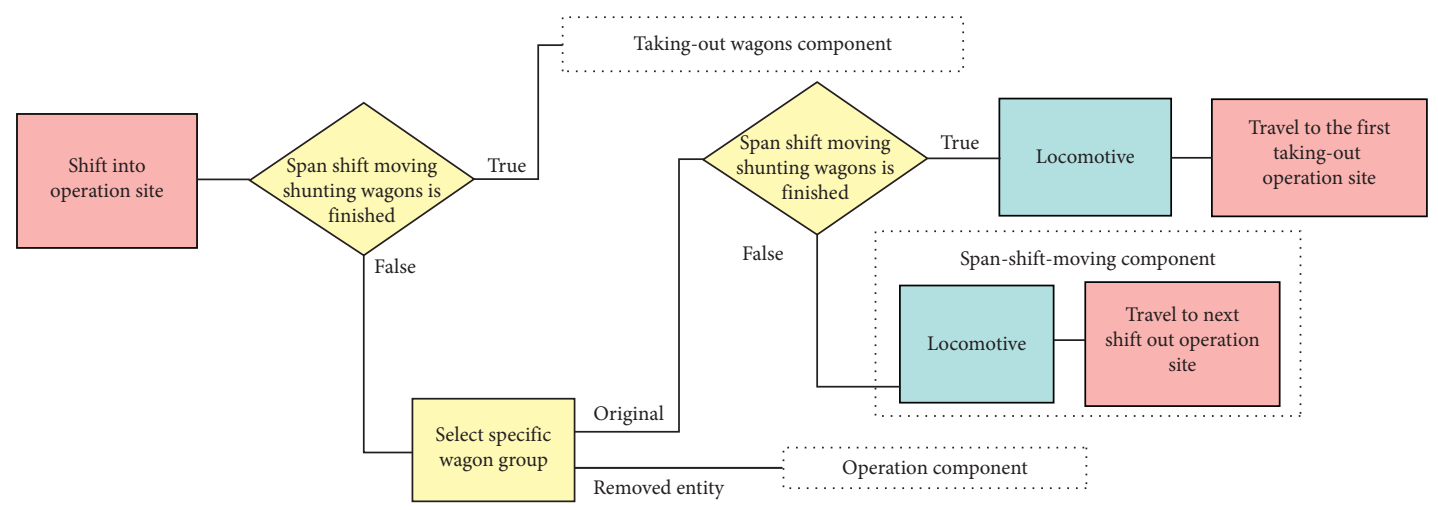

Figure 16: Flowchart of submodel for shift-into freight operation sites based on the Arena simulation platform.

shunting strategies of PTWS-SO and can analyze sensitivity of shunting strategies in the system. To depict a comprehensive picture for the PTWS-SO, the simulation results are analyzed in terms of shunting strategies, as presented in Section 6.2.

6.2.1. Monitoring the Output Factors. The output factors are important for monitoring the running status of PTWS-SO. They can expose the appearing failures of system in time. In this experiment, we use three output factors. The first one is the operation start time point of freight operation sites denoted as OSTP-FOS. The second one is the operation completion time point of freight operation sites denoted as OCTP-FOS. The third one is the taking-out time point of freight operation sites denoted as TOTPFOS. We observe the evolution of the output factors for whole planning horizon under different shunting strategies. Here, the dynamic change of output factors is constantly monitored at 24 hours of the day and night using the ARENA simulation platform. Table 2 and Figure 17 show the evolution of three output factors through 24 hours for PTWS-SO under the optimal configuration condition.
6.2.2. Depiction of Shunting Strategies. The placing-in sequence of freight operation sites is abbreviated as PIS-FOS. The taking-out sequence of freight operation sites is abbreviated as TOS-FOS. The shunting strategies of PTWS-SO can be observed in Figure 18.

6.2.3. Performance Evaluation. To evaluate the performance of the shunting strategies proposed, we use three measures of performance. The first one is the total detention time denoted as TDT. TDT is one of the major criterions in assessing the performance of the shunting strategy representing the total time which is composed of travel time between operation sites, loading/unloading time, and waiting time. The second measure of performance is the total waiting time denoted as TWT. TWT is another one of the major criterions representing the sum of the waiting time in all operation sites. The third measure of performance is the total travel time denoted as TTT. TTT represents the sum of the travel time between any pair of freight operation sites. The shunting strategies on PTWS-SO can be tested by the measures of performance and is, respectively, listed in Table 3. The results of comparison are displayed in Figure 19. 
TABLE 2: Output factors for shunting strategies on PTWS-SO.

\begin{tabular}{|c|c|c|c|c|c|c|c|c|c|}
\hline Site & Output factors & ATT-ECT & ADT-ECT & RTT-ECT & RDT-ECT & ATT-NL\&CT & ADT-NL\&CT & RTT-NL\&CT & RDT-NL\&CT \\
\hline \multirow{3}{*}{1} & OSTP-FOS & 7.634 & 12.167 & 7.634 & 12.501 & 7.634 & 12.167 & 7.634 & 12.501 \\
\hline & OCTP-FOS & 8.634 & 13.167 & 8.634 & 13.501 & 8.634 & 13.167 & 8.634 & 13.501 \\
\hline & TOTP-FOS & 12.434 & 14.967 & 11.567 & 15.834 & 13.367 & 14.967 & 12.501 & 14.167 \\
\hline \multirow{3}{*}{2} & OSTP-FOS & 7.834 & 11.967 & 7.834 & 10.567 & 7.834 & 11.967 & 7.834 & 10.567 \\
\hline & OCTP-FOS & 9.334 & 13.467 & 9.334 & 12.067 & 9.334 & 13.467 & 9.334 & 12.067 \\
\hline & TOTP-FOS & 12.634 & 15.167 & 11.767 & 12.701 & 13.567 & 15.167 & 12.701 & 12.701 \\
\hline \multirow{3}{*}{3} & OSTP-FOS & 8.801 & 8.934 & 7.934 & 8.934 & 8.801 & 8.934 & 7.934 & 8.934 \\
\hline & OCTP-FOS & 13.801 & 13.934 & 12.934 & 13.934 & 13.801 & 13.934 & 12.934 & 13.934 \\
\hline & TOTP-FOS & 15.667 & 15.267 & 14.801 & 16.134 & 15.667 & 15.267 & 14.801 & 14.467 \\
\hline \multirow{3}{*}{4} & OSTP-FOS & 8.267 & 10.134 & 8.467 & 10.134 & 8.267 & 10.134 & 8.467 & 10.134 \\
\hline & OCTP-FOS & 10.267 & 12.134 & 10.467 & 12.134 & 10.267 & 12.134 & 10.467 & 12.134 \\
\hline & TOTP-FOS & 13.067 & 13.934 & 12.201 & 13.134 & 12.334 & 12.534 & 11.467 & 13.134 \\
\hline \multirow{3}{*}{5} & OSTP-FOS & 9.467 & 9.601 & 8.601 & 9.601 & 9.467 & 9.601 & 8.601 & 9.601 \\
\hline & OCTP-FOS & 12.467 & 12.601 & 11.601 & 12.601 & 12.467 & 12.601 & 11.601 & 12.601 \\
\hline & TOTP-FOS & 13.061 & 14.467 & 12.734 & 15.334 & 12.867 & 13.067 & 12.001 & 13.267 \\
\hline \multirow{3}{*}{6} & OSTP-FOS & 9.801 & 9.934 & 8.934 & 9.934 & 9.801 & 9.934 & 8.934 & 9.934 \\
\hline & OCTP-FOS & 12.301 & 12.434 & 11.434 & 12.434 & 12.301 & 12.434 & 11.434 & 12.434 \\
\hline & TOTP-FOS & 13.267 & 14.134 & 12.401 & 13.334 & 12.534 & 12.734 & 11.667 & 13.601 \\
\hline \multirow{3}{*}{7} & OSTP-FOS & 11.501 & 10.967 & 10.634 & 11.567 & 11.501 & 10.967 & 10.634 & 11.567 \\
\hline & OCTP-FOS & 12.501 & 11.967 & 11.634 & 12.567 & 12.501 & 11.967 & 11.634 & 12.567 \\
\hline & TOTP-FOS & 14.567 & 13.101 & 13.701 & 14.367 & 14.567 & 14.034 & 13.701 & 15.567 \\
\hline \multirow{3}{*}{8} & OSTP-FOS & 10.601 & 8.067 & 9.734 & 8.067 & 10.601 & 8.067 & 9.734 & 8.067 \\
\hline & OCTP-FOS & 18.101 & 15.567 & 17.234 & 15.567 & 18.101 & 15.567 & 17.234 & 15.567 \\
\hline & TOTP-FOS & 18.967 & 16.434 & 18.101 & 17.001 & 18.967 & 16.434 & 18.101 & 16.467 \\
\hline
\end{tabular}

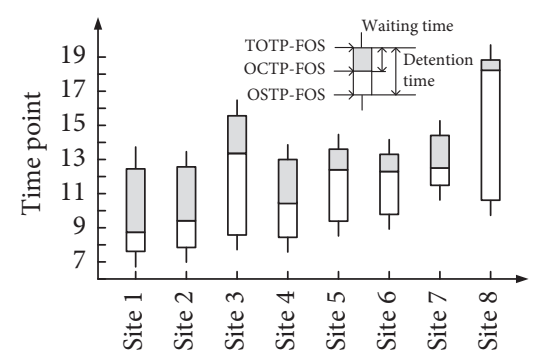

(a)

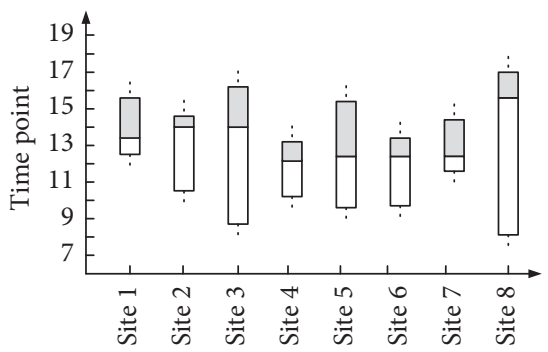

(d)

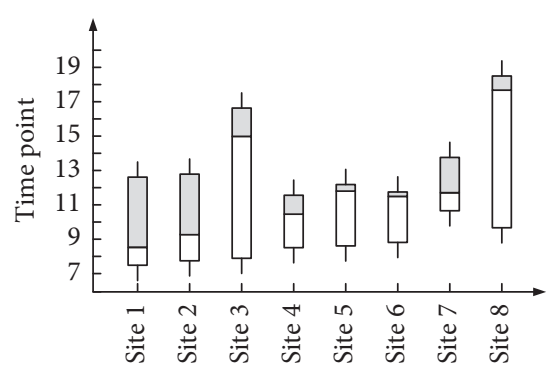

(g)

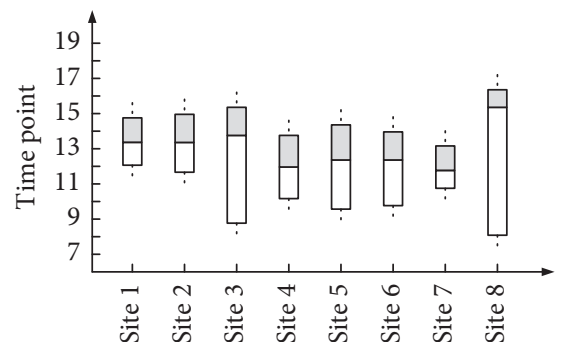

(b)

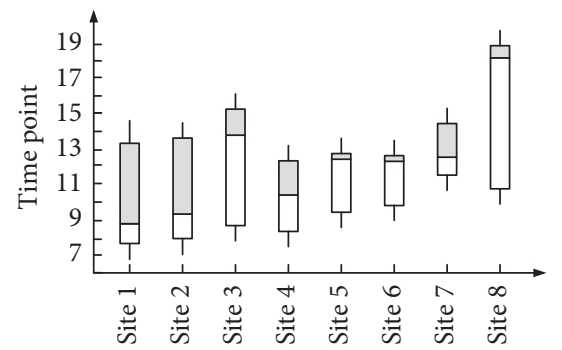

(e)

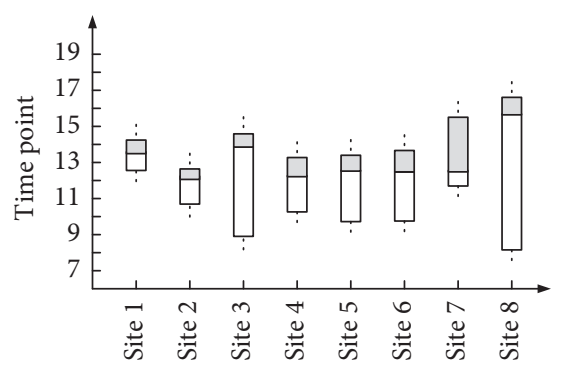

(h)

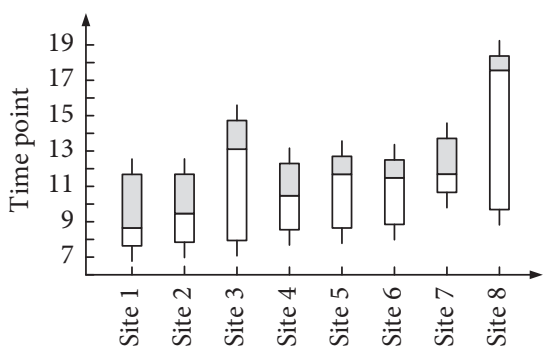

(c)

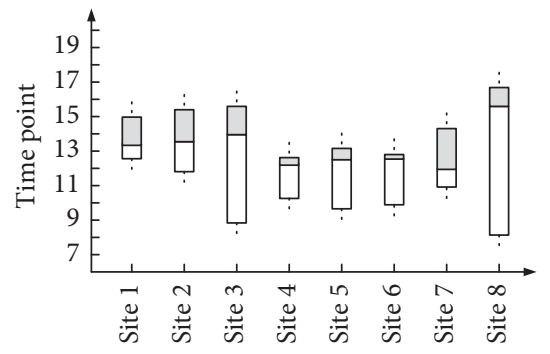

(f)

FIGURe 17: Output factors for shunting strategies on PTWS-SO: (a) ATT-ECT strategy; (b) ADT-ECT strategy; (c) RTT-ECT strategy; (d) RDT-ECT strategy; (e) ATT-NL\&CT strategy; (f) ADT-NL\&CT strategy; (g) RTT-NL\&CT strategy; (h) RDT-NL\&CT strategy. 


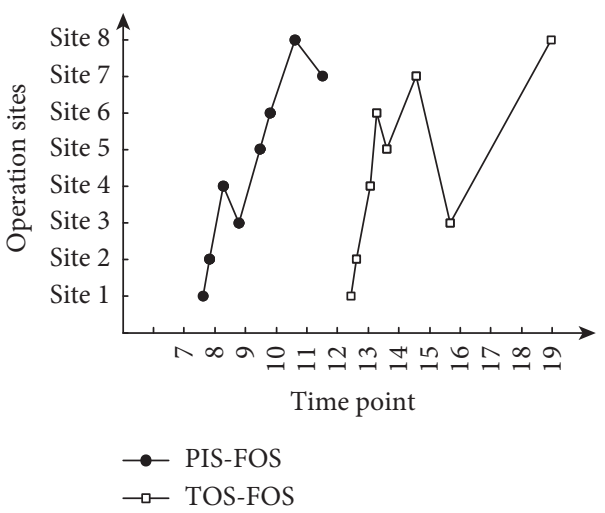

(a)

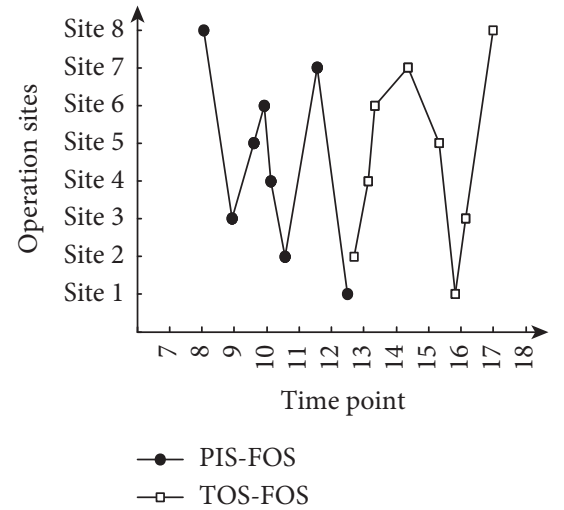

(d)

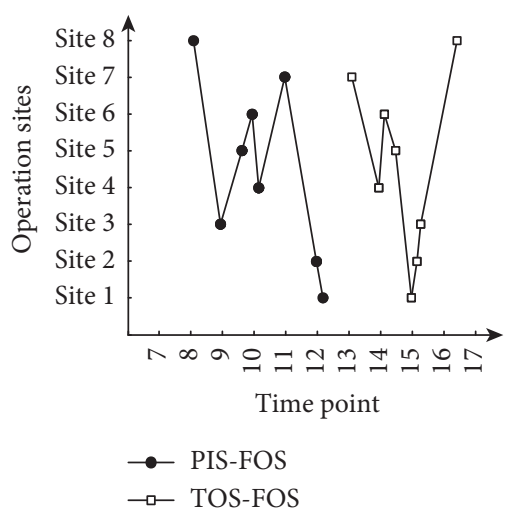

(b)

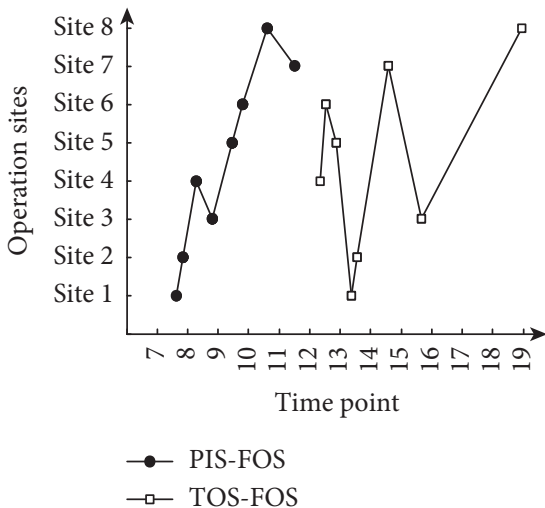

(e)

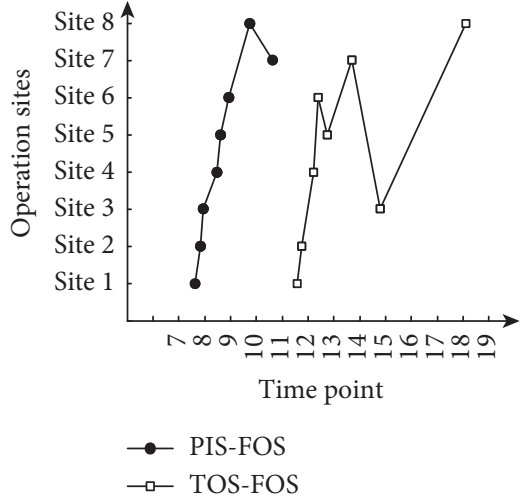

(c)

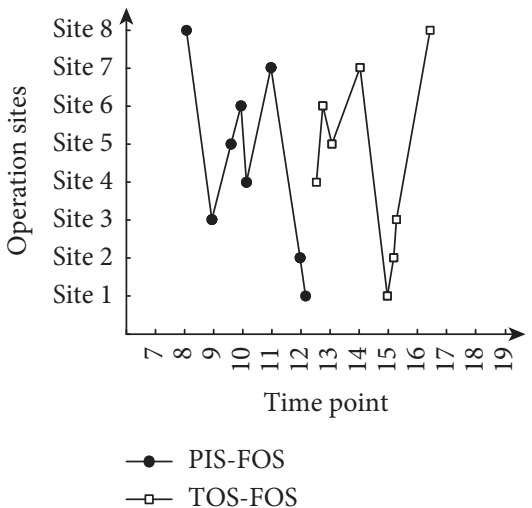

(f)

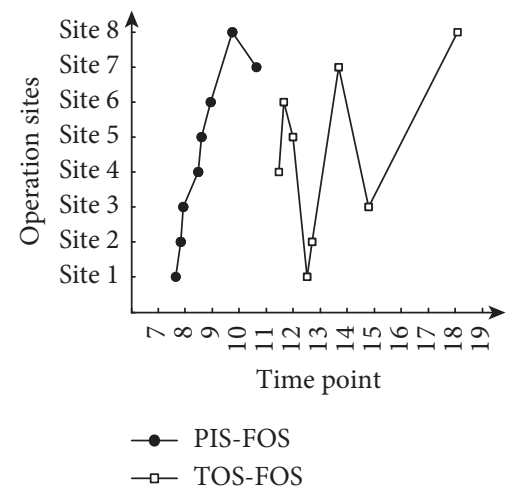

(g)

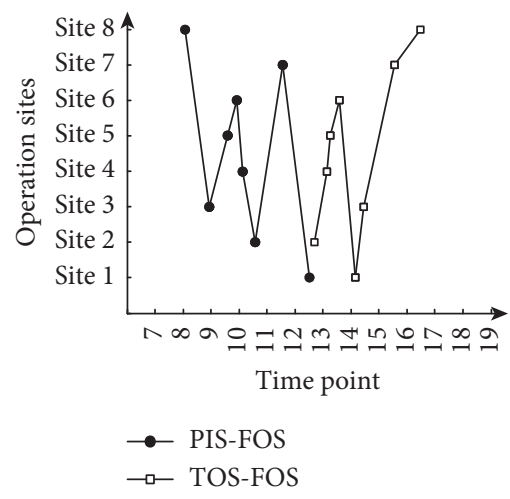

(h)

FIGURE 18: Placing-in and taking-out shunting scheme under different shunting strategies: (a) ATT-ECT strategy; (b) ADT-ECT strategy; (c) RTT-ECT strategy; (d) RDT-ECT strategy; (e) ATT-NL\&CT strategy; (f) ADT-NL\&CT strategy; (g) RTT-NL\&CT strategy; (h) RDT-NL\&CT strategy.

For the PTWS-SO problem, the performance of the RDT-NL\&CT shunting strategy is obviously prominent when compared to other shunting strategies. When the major criterion TDT is contrasted, the difference is very clear. The RDT-NL\&CT shunting strategy can generate fewer total detention time than other any shunting strategy. Meanwhile, the superiority of the RDT-NL\&CT shunting strategy in analytical indicator criterion TWT is practically obviously. Furthermore, RDT-NL\&CT shunting strategy maintains a good performance in another analytical indicator TTT. In sum, we can conclude that the RDT-NL\&CT shunting strategy is effective to obtain good placing-in and taking-out freight operation sites sequence for the PTWS-SO problem. In addition, the results obtained indicate that the ADT-NL\&CT shunting strategy is also acceptable for practical applications.

6.3. Testing Performance of PTWS-SSMS. The branch-shaped freight operation network used to test PTWS-SSMS is same 
TABle 3: Performance for different shunting strategies on PTWS-SO.

\begin{tabular}{|c|c|c|c|c|c|c|c|c|c|}
\hline Site & Measure & ATT-ECT & ADT-ECT & RTT-ECT & RDT-ECT & ATT-NL\&CT & ADT-NL\&CT & RTT-NL\&CT & RDT-NL\&CT \\
\hline \multirow{2}{*}{1} & $\mathrm{WT}_{1}{ }^{*}$ & 3.800 & 1.800 & 2.933 & 2.333 & 4.733 & 1.800 & 3.867 & 0.666 \\
\hline & $\mathrm{DT}_{1}{ }^{*}$ & 4.800 & 2.800 & 3.933 & 3.333 & 5.733 & 2.800 & 4.867 & 1.666 \\
\hline \multirow[b]{2}{*}{2} & $\mathrm{WT}_{2}{ }^{*}$ & 2.700 & 1.700 & 2.433 & 0.634 & 3.633 & 1.700 & 3.367 & 0.634 \\
\hline & $\mathrm{DT}_{2}{ }^{*}$ & 4.800 & 3.200 & 3.933 & 2.134 & 5.733 & 3.200 & 4.867 & 2.134 \\
\hline \multirow{2}{*}{3} & $\mathrm{WT}_{3}{ }^{*}$ & 1.866 & 1.333 & 1.867 & 2.200 & 1.866 & 1.333 & 1.867 & 0.533 \\
\hline & $\mathrm{DT}_{3}{ }^{*}$ & 6.866 & 6.333 & 6.867 & 7.200 & 6.866 & 6.333 & 6.867 & 5.533 \\
\hline \multirow[b]{2}{*}{4} & $\mathrm{WT}_{4}{ }^{*}$ & 2.800 & 1.800 & 1.734 & 1.000 & 2.067 & 0.400 & 1.000 & 1.000 \\
\hline & $\mathrm{DT}_{4}{ }^{*}$ & 4.800 & 3.800 & 3.734 & 3.00 & 4.067 & 2.400 & 3.00 & 3.000 \\
\hline \multirow{2}{*}{5} & $\mathrm{WT}_{5}{ }^{*}$ & 1.134 & 1.866 & 1.133 & 2.733 & 0.400 & 0.466 & 0.400 & 0.666 \\
\hline & $\mathrm{DT}_{5}{ }^{*}$ & 4.134 & 4.866 & 4.133 & 5.733 & 3.400 & 3.466 & 3.400 & 3.666 \\
\hline \multirow{2}{*}{6} & $\mathrm{WT}_{6}{ }^{*}$ & 0.966 & 1.700 & 0.967 & 0.900 & 0.233 & 0.300 & 0.233 & 1.667 \\
\hline & $\mathrm{DT}_{6}{ }^{*}$ & 3.466 & 4.200 & 3.467 & 3.400 & 2.733 & 2.800 & 2.733 & 3.667 \\
\hline \multirow{2}{*}{7} & $\mathrm{WT}_{7}{ }^{*}$ & 2.066 & 1.134 & 2.067 & 1.800 & 2.066 & 2.067 & 2.067 & 3.000 \\
\hline & $\mathrm{DT}_{7}{ }^{*}$ & 3.066 & 2.134 & 3.067 & 2.800 & 3.066 & 3.067 & 3.067 & 4.000 \\
\hline \multirow{2}{*}{8} & $\mathrm{WT}_{8}{ }^{*}$ & 0.866 & 0.867 & 0.867 & 1.434 & 0.866 & 0.867 & 0.867 & 0.900 \\
\hline & $\mathrm{DT}_{8}{ }^{*}$ & 8.366 & 8.367 & 8.367 & 8.934 & 8.366 & 8.367 & 8.367 & 8.400 \\
\hline & TWT & 16.198 & 12.200 & 14.001 & 13.034 & 15.864 & 8.933 & 13.668 & 8.566 \\
\hline & TDT & 40.298 & 35.700 & 37.501 & 36.534 & 39.964 & 32.433 & 37.168 & 32.066 \\
\hline & TTT & 9.066 & 8.666 & 8.733 & 9.533 & 9.066 & 8.666 & 8.200 & 8.366 \\
\hline
\end{tabular}

${ }^{*} \mathrm{WT}_{i}$ is the waiting time in freight operation site $i \in A . \mathrm{DT}_{i}$ is the total time which is spent on loading/unloading and waiting in freight operation site $i \in A$.

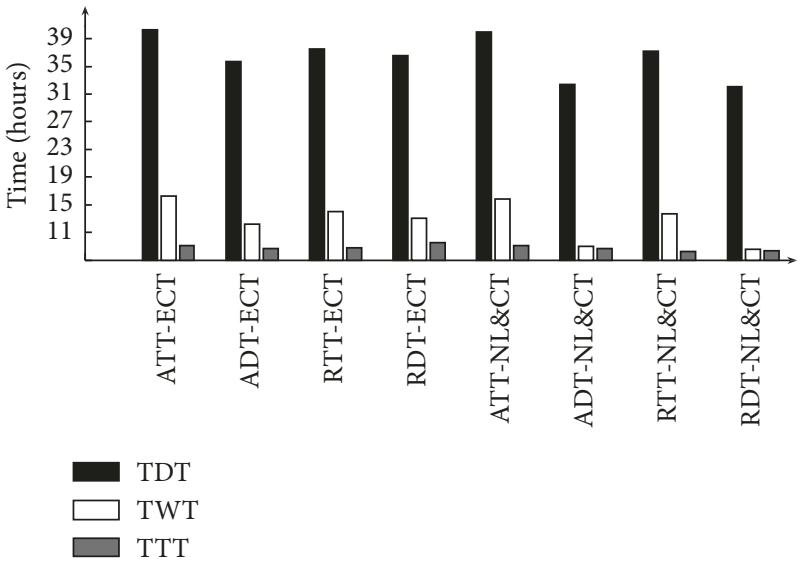

FIGURE 19: Results of comparison for different shunting strategies on PTWS-SO.

with that of PTWS-SO. But there are 3 shift-out freight operation sites. That is to say, the local wagons decoupled from inbound trains include 3 groups of double operation wagons. After the 3 groups of double operation wagon are detached and have finished the unloading at the shift-out freight operation sites, they will be moved to another freight site for loading.

Under scenarios of PTWS-SSMS, there are two spanshift-moving strategies, i.e., SSM-ECT and SSM-NL\&CT. Here, we take the two span-shift-moving strategies into the existing shunting strategies and generate sixteen hybrid shunting strategies. And then, we use the ARENA platform to simulate sixteen hybrid shunting strategies.

We observe the evolution of the output factors for whole planning horizon under sixteen hybrid shunting strategies. Tables 4 and 5 and Figure 20 show the evolution of the output factors through 24 hours for PTWS-SSMS.
There are two numbers in the cell of the table. The number on the left of cell represents output factors under SSM-ECT. The number on the right of cell represents output factors under SSM-NL\&CT.

The span-shift-moving sequence of freight operation sites is abbreviated as SSMS-FOS. The hybrid shunting strategies of PTWS-SSMS can be observed in Figure 21. All the local operation wagons are required to dispatch to relevant shift-in freight sites, and the locomotive is not necessary to go back to shift-out freight sites when picking wagons up.

The hybrid shunting strategies of PTWS-SSMS can be tested by the measures of performance and is, respectively, listed in Tables 6 and 7. The results of comparison are displayed in Figure 22.

There are two numbers in the cell of the table. The number on the left of cell represents performance measures 
TABLE 4: Output factors for shunting strategies on PTWS-SSMS under SSM-ECT.

\begin{tabular}{|c|c|c|c|c|c|c|c|c|c|}
\hline Site & Output factors & ATT-ECT & ADT-ECT & RTT-ECT & RDT-ECT & ATT-NL\&CT & ADT-NL\&CT & RTT-NL\&CT & RDT-NL\&CT \\
\hline 1 & TOTP-FOS & 17.301 & 19.167 & 16.434 & 18.434 & 17.301 & 19.234 & 16.434 & 17.501 \\
\hline \multirow[t]{2}{*}{$2^{*}$} & TOTP-FOS & 12.501 & 16.501 & 11.634 & 12.701 & 12.501 & 16.501 & 11.634 & 12.701 \\
\hline & OSTP-FOS & 7.834 & 11.967 & 7.834 & 10.567 & 7.834 & 11.967 & 7.834 & 10.567 \\
\hline \multirow[t]{2}{*}{$9^{\#}$} & OCTP-FOS & 14.901 & 18.901 & 14.034 & 15.101 & 14.401 & 18.901 & 14.034 & 15.101 \\
\hline & TOTP-FOS & 19.867 & 21.867 & 19.001 & 19.734 & 20.934 & 21.867 & 20.067 & 20.134 \\
\hline 3 & TOTP-FOS & 18.867 & 19.467 & 18.001 & 18.734 & 17.601 & 19.534 & 16.734 & 17.801 \\
\hline \multirow[t]{2}{*}{$4^{*}$} & TOTP-FOS & 14.134 & 14.934 & 13.267 & 14.334 & 14.134 & 14.934 & 13.267 & 14.334 \\
\hline & OSTP-FOS & 8.267 & 10.134 & 8.467 & 10.134 & 8.267 & 10.134 & 8.467 & 10.134 \\
\hline \multirow[t]{2}{*}{$10^{\#}$} & OCTP-FOS & 16.834 & 17.634 & 15.967 & 17.034 & 16.334 & 17.634 & 15.967 & 17.034 \\
\hline & TOTP-FOS & 20.301 & 21.434 & 19.434 & 21.301 & 20.501 & 21.434 & 19.634 & 19.701 \\
\hline 5 & TOTP-FOS & 18.201 & 18.667 & 17.334 & 17.934 & 18.267 & 20.201 & 17.401 & 18.467 \\
\hline 6 & TOTP-FOS & 17.867 & 18.334 & 17.001 & 17.601 & 18.601 & 20.534 & 17.734 & 18.801 \\
\hline \multirow[t]{2}{*}{$7^{*}$} & TOTP-FOS & 15.367 & 13.101 & 14.501 & 15.567 & 15.367 & 13.101 & 14.501 & 15.567 \\
\hline & OSTP-FOS & 11.501 & 10.967 & 10.634 & 11.567 & 11.501 & 10.967 & 10.634 & 11.567 \\
\hline \multirow[t]{2}{*}{$11^{\#}$} & OCTP-FOS & 17.434 & 15.167 & 16.567 & 17.634 & 16.934 & 15.167 & 16.567 & 17.634 \\
\hline & TOTP-FOS & 21.234 & 20.501 & 20.367 & 22.234 & 19.567 & 18.367 & 19.634 & 21.101 \\
\hline 8 & TOTP-FOS & 21.401 & 20.667 & 20.534 & 20.534 & 19.401 & 18.201 & 18.534 & 16.801 \\
\hline
\end{tabular}

Note. ${ }^{*}$ The freight operation site is shift-out site; ${ }^{\#}$ the freight operation site is shift-into site.

TABLE 5: Output factors for shunting strategies on PTWS-SSMS under SSM-NL\&CT.

\begin{tabular}{|c|c|c|c|c|c|c|c|c|c|}
\hline Site & Output factors & ATT-ECT & ADT-ECT & RTT-ECT & RDT-ECT & ATT-NL\&CT & ADT-NL\&CT & RTT-NL\&CT & RDT-NL\&CT \\
\hline 1 & TOTP-FOS & 17.301 & 18.434 & 16.434 & 18.101 & 17.701 & 18.901 & 16.834 & 18.634 \\
\hline \multirow[t]{2}{*}{$2^{*}$} & TOTP-FOS & 15.567 & 15.767 & 14.701 & 12.701 & 15.567 & 15.767 & 14.701 & 12.701 \\
\hline & OSTP-FOS & 7.834 & 11.967 & 7.834 & 10.567 & 7.834 & 11.967 & 7.834 & 10.567 \\
\hline \multirow[t]{2}{*}{$9^{\#}$} & OCTP-FOS & 17.967 & 18.167 & 17.101 & 15.101 & 17.967 & 18.167 & 17.101 & 15.101 \\
\hline & TOTP-FOS & 21.734 & 21.601 & 20.867 & 19.401 & 20.934 & 21.134 & 20.067 & 16.801 \\
\hline 3 & TOTP-FOS & 18.867 & 18.734 & 18.001 & 18.401 & 18.001 & 19.201 & 17.134 & 18.934 \\
\hline \multirow[t]{2}{*}{$4^{*}$} & TOTP-FOS & 12.334 & 12.534 & 11.467 & 15.667 & 12.334 & 12.534 & 11.467 & 15.667 \\
\hline & OSTP-FOS & 8.267 & 10.134 & 8.467 & 10.134 & 8.267 & 10.134 & 8.467 & 10.134 \\
\hline \multirow[t]{2}{*}{$10^{\#}$} & OCTP-FOS & 15.034 & 15.234 & 14.167 & 18.367 & 15.034 & 15.234 & 14.167 & 18.367 \\
\hline & TOTP-FOS & 19.834 & 19.701 & 18.967 & 21.301 & 16.901 & 17.101 & 16.034 & 20.834 \\
\hline 5 & TOTP-FOS & 18.201 & 17.934 & 17.334 & 17.601 & 18.667 & 19.867 & 17.801 & 19.601 \\
\hline 6 & TOTP-FOS & 17.867 & 17.601 & 17.001 & 17.267 & 19.001 & 20.201 & 18.134 & 19.934 \\
\hline \multirow[t]{2}{*}{$7^{*}$} & TOTP-FOS & 13.567 & 13.767 & 12.701 & 13.834 & 13.567 & 13.767 & 12.701 & 13.834 \\
\hline & OSTP-FOS & 11.501 & 10.967 & 10.634 & 11.567 & 11.501 & 10.967 & 10.634 & 11.567 \\
\hline \multirow[t]{2}{*}{$11^{\#}$} & OCTP-FOS & 15.634 & 15.834 & 14.767 & 15.901 & 15.634 & 15.834 & 14.767 & 15.901 \\
\hline & TOTP-FOS & 20.767 & 20.634 & 19.901 & 20.367 & 19.967 & 18.034 & 19.101 & 17.767 \\
\hline 8 & TOTP-FOS & 22.534 & 20.467 & 21.667 & 20.201 & 19.801 & 17.867 & 18.934 & 17.601 \\
\hline
\end{tabular}

Note. ${ }^{*}$ The freight operation site is shift-out site; ${ }^{\#}$ the freight operation site is shift-into site.

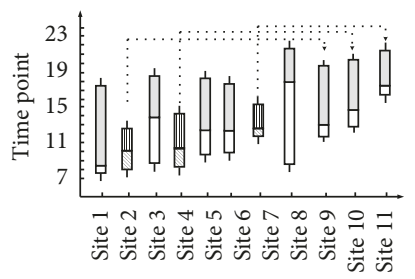

(a)

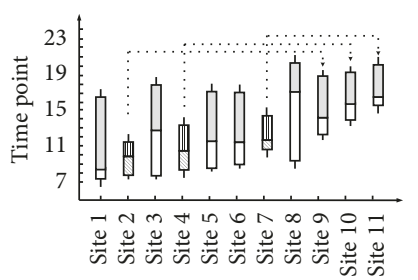

(e)

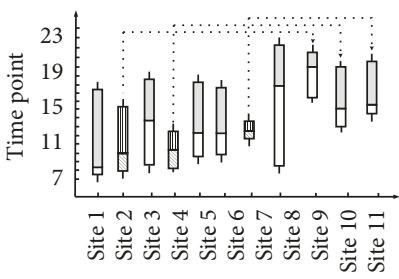

(b)

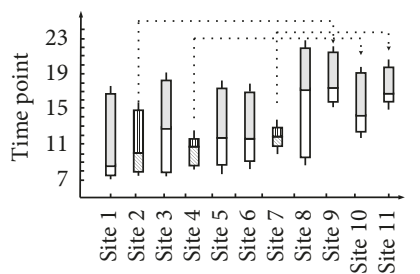

(f)

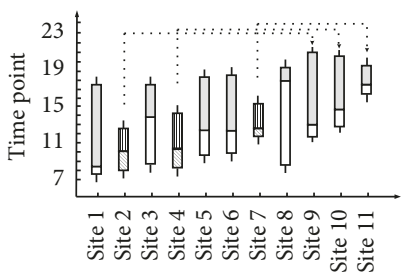

(c)

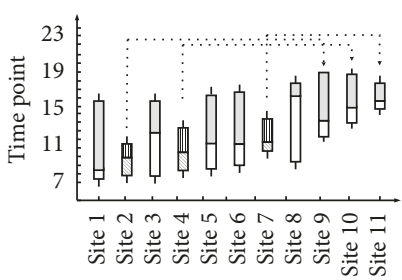

(g)

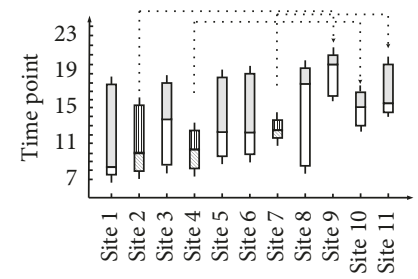

(d)

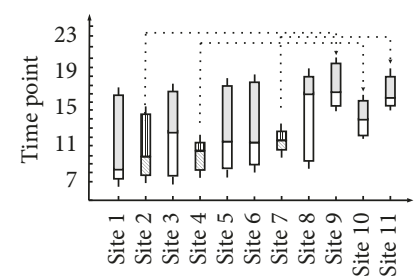

(h)

Figure 20: Continued. 


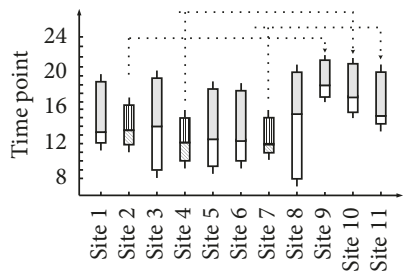

(i)

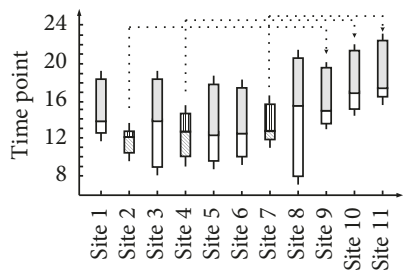

(m)

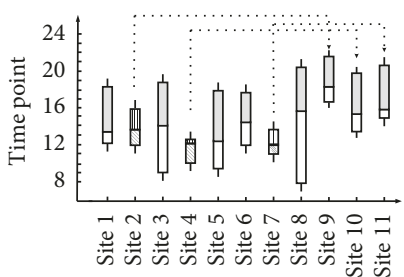

(j)

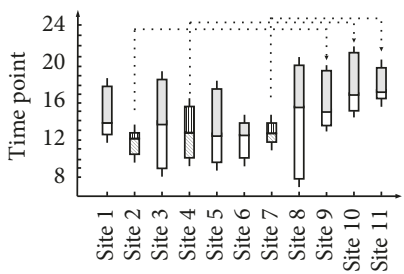

(n)

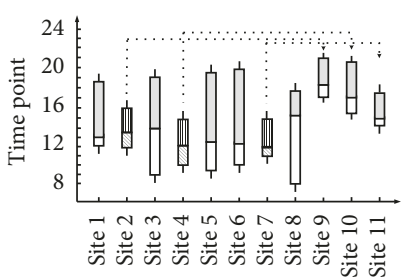

(k)

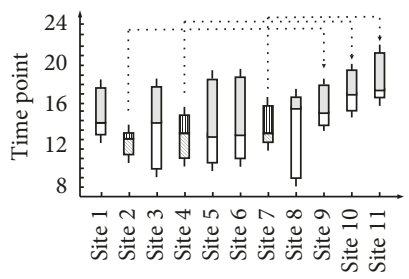

(o)

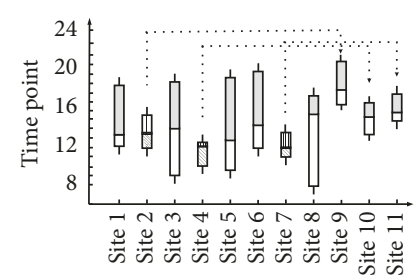

(l)

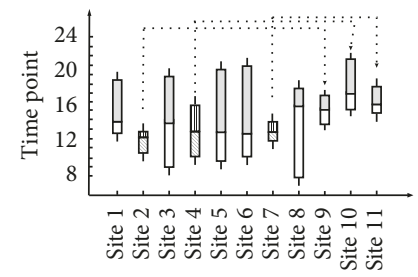

(p)

FIGURE 20: Output factors for shunting strategies on PTWS-SSMS: (a) ATT-ECT strategy under SSM-ECT; (b) ATT-ECT strategy under SSM-NL\&CT; (c) ATT-NL\&CT strategy under SSM-ECT; (d) ATT-NL\&CT strategy under SSM-NL\&CT; (e) RTT-ECT strategy under SSM-ECT; (f) RTT-ECT strategy under SSM-NL\&CT; (g) RTT-NL\&CT strategy under SSM-ECT; (h) RTT-NL\&CT strategy under SSMNL\&CT; (i) ADT-ECT strategy under SSM-ECT; (j) ADT-ECT strategy under SSM-NL\&CT; (k) ADT-NL\&CT strategy under SSM-ECT; (l) ADT-NL\&CT strategy under SSM-NL\&CT; (m) RDT-ECT strategy under SSM-ECT; (n) RDT-ECT strategy under SSM-NL\&CT; (o) RDTNL\&CT strategy under SSM-ECT; (p) RDT-NL\&CT strategy under SSM-NL\&CT.

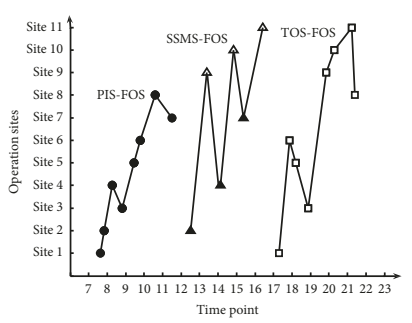

$\Delta$ Shift-out site
$\Delta$ Shift-into site

(a)

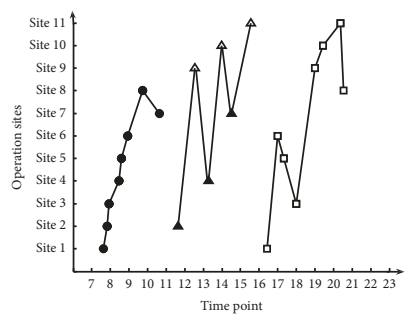

$\Delta$ Shift-out site
$\Delta$ Shift-into site

(e)

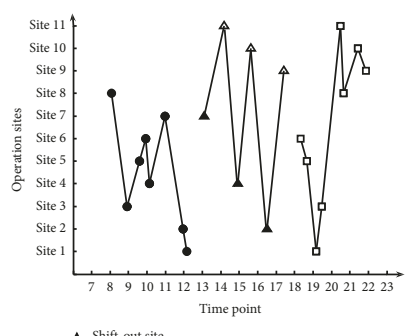

S Shift-out site
$\Delta$ Shift-into site

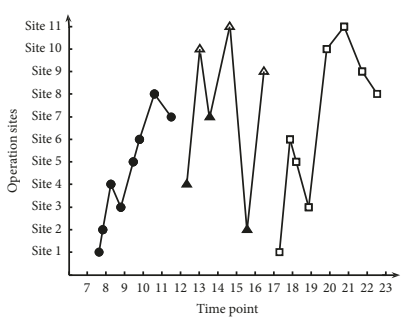

$\stackrel{\Delta \text { Shifit-out site }}{\Delta \text { Shiftinto site }}$

(b)

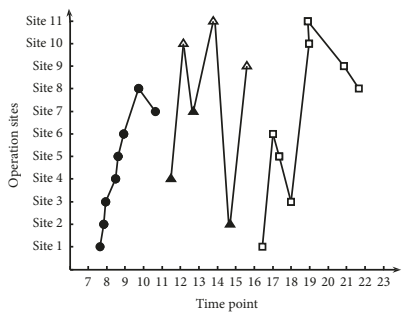

$\Delta$ Shift-out site
$\Delta$ Shift-into site

(f)

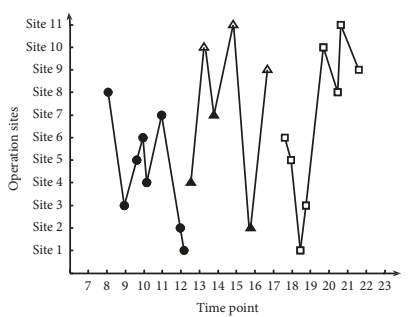

$\Delta$ Shift-out site
$\Delta$ Shift-into site

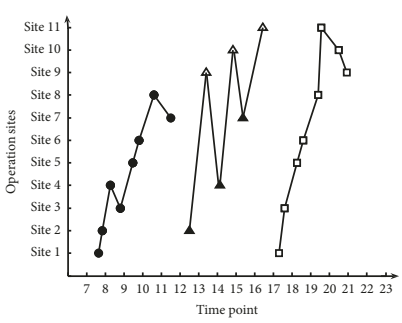

$\Delta$ Shift-out site
$\Delta$ Shift-into site

(c)

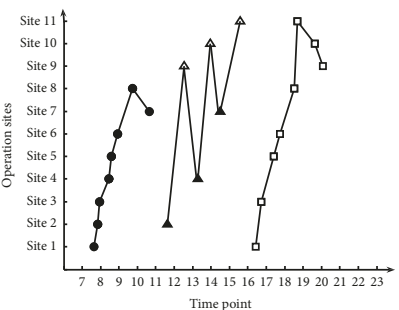

$\Delta$ Shift-out site
$\Delta$ Shift-into site

(g)

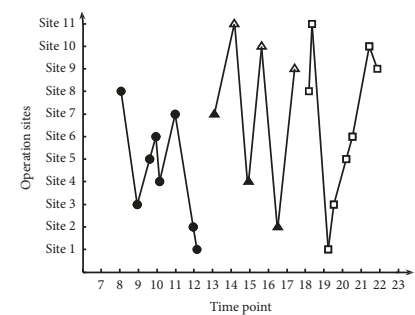

S Shift-out site
$\Delta$ Shift-into site

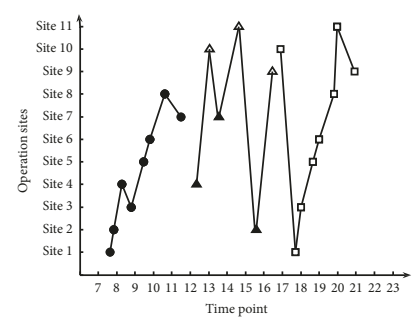

Shift-out site
Shift-into site

(d)

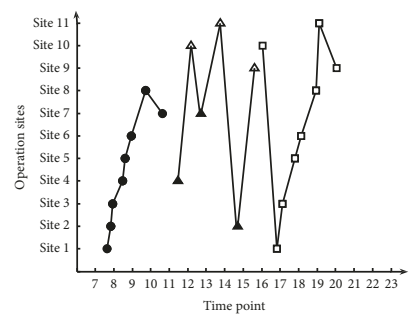

$\Delta$ Shift-out site
$\Delta$ Shift-into site

(h)

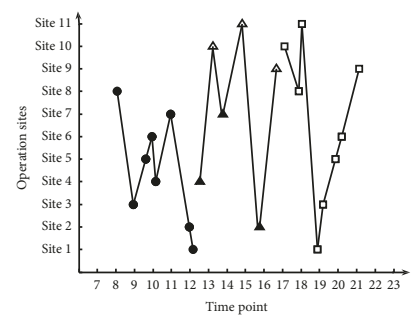

S Shift-out site
$\Delta$ Shift-into site

(i)

(j)

(k)

(l)

Figure 21: Continued. 


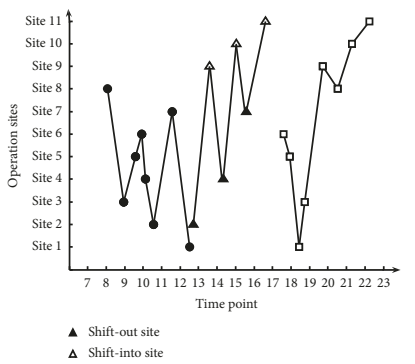

$(\mathrm{m})$

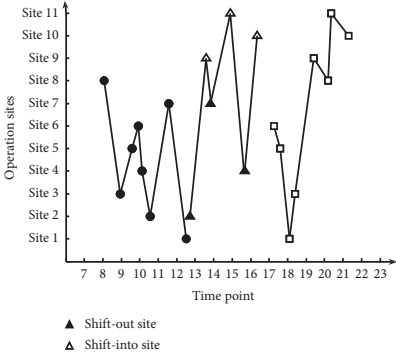

(n)

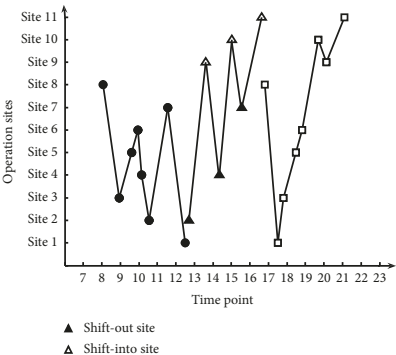

(o)

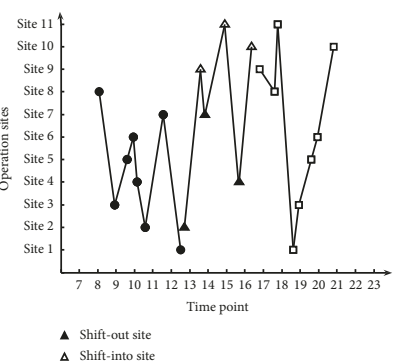

(p)

FIGURE 21: Hybrid shunting scheme under different shunting strategies: (a) ATT-ECT strategy under SSM-ECT; (b) ATT-ECT strategy under SSM-NL\&CT; (c) ATT-NL\&CT strategy under SSM-ECT; (d) ATT-NL\&CT strategy under SSM-NL\&CT; (e) RTT-ECT strategy under SSM-ECT; (f) RTT-ECT strategy under SSM-NL\&CT; (g) RTT-NL\&CT strategy under SSM-ECT; (h) RTT-NL\&CT strategy under SSM-NL\&CT; (i) ADT-ECT strategy under SSM-ECT; (j) ADT-ECT strategy under SSM-NL\&CT; (k) ADT-NL\&CT strategy under SSMECT; (l) ADT-NL\&CT strategy under SSM-NL\&CT; (m) RDT-ECT strategy under SSM-ECT; (n) RDT-ECT strategy under SSM-NL\&CT; (o) RDT-NL\&CT strategy under SSM-ECT; (p) RDT-NL\&CT strategy under SSM-NL\&CT.

TABle 6: Performance for different shunting strategies on PTWS under SSM-ECT.

\begin{tabular}{|c|c|c|c|c|c|c|c|c|c|}
\hline Site & Measure & ATT-ECT & ADT-ECT & RTT-ECT & RDT-ECT & ATT-NL\&CT & ADT-NL\&CT & RTT-NL\&CT & RDT-NL\&CT \\
\hline \multirow{2}{*}{1} & $\mathrm{WT}_{1}$ & 8.667 & 6.000 & 7.800 & 4.933 & 8.667 & 6.067 & 7.800 & 4.000 \\
\hline & $\mathrm{DT}_{1}$ & 9.667 & 7.000 & 8.800 & 5.933 & 9.667 & 7.067 & 8.800 & 5.000 \\
\hline \multirow{2}{*}{$2^{*}$} & $\mathrm{WT}_{2}$ & 2.567 & 3.034 & 2.300 & 0.634 & 2.567 & 3.034 & 2.300 & 0.634 \\
\hline & $\mathrm{DT}_{2}$ & 4.667 & 4.534 & 3.800 & 2.134 & 4.667 & 4.534 & 3.800 & 2.134 \\
\hline \multirow{2}{*}{3} & $\mathrm{WT}_{3}$ & 5.066 & 5.533 & 5.067 & 4.800 & 3.800 & 5.600 & 3.800 & 3.867 \\
\hline & $\mathrm{DT}_{3}$ & 10.066 & 10.533 & 10.067 & 9.800 & 8.800 & 10.600 & 8.800 & 8.867 \\
\hline \multirow{2}{*}{$4^{*}$} & $\mathrm{WT}_{4}$ & 3.867 & 2.800 & 2.800 & 2.200 & 3.867 & 2.800 & 2.800 & 2.200 \\
\hline & $\mathrm{DT}_{4}$ & 5.867 & 4.800 & 4.800 & 4.200 & 5.867 & 4.800 & 4.800 & 4.200 \\
\hline \multirow{2}{*}{5} & $\mathrm{WT}_{5}$ & 5.734 & 6.066 & 5.733 & 5.333 & 5.800 & 7.600 & 5.800 & 5.866 \\
\hline & $\mathrm{DT}_{5}$ & 8.734 & 9.066 & 8.733 & 8.333 & 8.800 & 10.600 & 8.800 & 8.866 \\
\hline \multirow{2}{*}{$6^{*}$} & $\mathrm{WT}_{6}$ & 5.566 & 5.900 & 5.567 & 5.167 & 6.300 & 8.100 & 6.300 & 6.367 \\
\hline & $\mathrm{DT}_{6}$ & 8.066 & 8.400 & 8.067 & 7.667 & 8.800 & 10.600 & 8.800 & 8.867 \\
\hline \multirow{2}{*}{7} & $\mathrm{WT}_{7}$ & 2.866 & 1.134 & 2.867 & 3.000 & 2.866 & 1.134 & 2.867 & 3.000 \\
\hline & $\mathrm{DT}_{7}$ & 3.866 & 2.134 & 3.867 & 4.000 & 3.866 & 2.134 & 3.867 & 4.000 \\
\hline \multirow{2}{*}{8} & $\mathrm{WT}_{8}$ & 3.300 & 5.100 & 3.300 & 4.967 & 1.300 & 2.634 & 1.300 & 1.234 \\
\hline & $\mathrm{DT}_{8}$ & 10.800 & 12.600 & 10.800 & 12.467 & 8.800 & 10.134 & 8.800 & 8.734 \\
\hline \multirow{2}{*}{$9^{\#}$} & $\mathrm{WT}_{9}$ & 4.966 & 2.966 & 4.967 & 4.633 & 6.533 & 2.966 & 6.033 & 5.033 \\
\hline & $\mathrm{DT}_{9}$ & 6.466 & 4.466 & 6.467 & 6.133 & 7.533 & 4.466 & 7.533 & 6.533 \\
\hline \multirow{2}{*}{$10^{\#}$} & $\mathrm{WT}_{10}$ & 3.467 & 3.8 & 3.467 & 4.267 & 4.167 & 3.800 & 3.667 & 2.667 \\
\hline & $\mathrm{DT}_{10}$ & 5.467 & 5.8 & 5.467 & 6.267 & 5.667 & 5.800 & 5.667 & 4.667 \\
\hline \multirow{2}{*}{$11^{\#}$} & $\mathrm{WT}_{11}$ & 3.8 & 5.334 & 3.8 & 4.600 & 2.633 & 3.200 & 2.134 & 3.467 \\
\hline & $\mathrm{DT}_{11}$ & 4.8 & 6.334 & 4.8 & 5.600 & 3.133 & 4.200 & 3.134 & 4.467 \\
\hline \multirow{3}{*}{\multicolumn{2}{|c|}{$\begin{array}{l}\text { TWT } \\
\text { TDT } \\
\text { TTT }\end{array}$}} & 49.866 & 47.667 & 47.668 & 44.534 & 48.5 & 46.935 & 44.801 & 38.335 \\
\hline & & 78.466 & 75.667 & 75.668 & 72.534 & 75.6 & 74.935 & 72.801 & 66.335 \\
\hline & & 15.1 & 14.733 & 14.733 & 17.033 & 13.166 & 14.733 & 12.833 & 13.966 \\
\hline
\end{tabular}

Note. ${ }^{*}$ The freight operation site is shift-out site; ${ }^{\#}$ the freight operation site is shift-into site.

under SSM-ECT. The number on the right of cell represents performance measures under SSM-NL\&CT.

For the PTWS-SSMS problem, the performance of shunting strategies under SSM-NL\&CT is more prominent than that of shunting strategies under SSM-ECT in generally. Under SSM-NL\&CT, the performance of RDT-NL\&CT and ADT-NL\&CT strategy are better than others when the major criterion TDT is contrasted. Moreover, they can create less total waiting time than other any shunting strategy in analytical indicator criterion TWT. Furthermore, a good performance is maintained for them in another analytical indicator TTT. In summary, we can conclude that the RDTNL\&CT and ADT-NL\&CT shunting strategy under SSMNL\&CT are effective to obtain good placing-in and takingout freight operation sites sequence for the PTWS-SSMS problem. 
TABLE 7: Performance for different shunting strategies on PTWS under SSM-NL\&CT.

\begin{tabular}{|c|c|c|c|c|c|c|c|c|c|}
\hline Site & Measure & ATT-ECT & ADT-ECT & RTT-ECT & RDT-ECT & ATT-NL\&CT & ADT-NL\&CT & RTT-NL\&CT & RDT-NL\&CT \\
\hline \multirow{2}{*}{1} & $\mathrm{WT}_{1}$ & 8.667 & 5.267 & 7.800 & 4.600 & 9.067 & 5.734 & 8.200 & 5.133 \\
\hline & $\mathrm{DT}_{1}$ & 9.667 & 6.267 & 8.800 & 5.600 & 10.067 & 6.734 & 9.200 & 6.133 \\
\hline \multirow{2}{*}{$2^{*}$} & $\mathrm{WT}_{2}$ & 5.633 & 2.300 & 5.367 & 0.634 & 5.633 & 2.300 & 5.367 & 0.634 \\
\hline & $\mathrm{DT}_{2}$ & 7.733 & 3.800 & 6.867 & 2.134 & 7.733 & 3.800 & 6.867 & 2.134 \\
\hline \multirow{2}{*}{3} & $\mathrm{WT}_{3}$ & 5.066 & 4.800 & 5.067 & 4.467 & 4.200 & 5.267 & 4.200 & 5.000 \\
\hline & $\mathrm{DT}_{3}$ & 10.066 & 9.800 & 10.067 & 9.467 & 9.200 & 10.267 & 9.200 & 10.000 \\
\hline \multirow{2}{*}{$4^{*}$} & $\mathrm{WT}_{4}$ & 2.067 & 0.400 & 1.000 & 3.533 & 2.067 & 0.400 & 1.000 & 3.533 \\
\hline & $\mathrm{DT}_{4}$ & 4.067 & 2.400 & 3.000 & 5.533 & 4.067 & 2.400 & 3.000 & 5.533 \\
\hline \multirow{2}{*}{5} & $\mathrm{WT}_{5}$ & 5.734 & 5.333 & 5.733 & 5.000 & 6.200 & 7.266 & 6.200 & 7.000 \\
\hline & $\mathrm{DT}_{5}$ & 8.734 & 8.333 & 8.733 & 8.000 & 9.200 & 10.266 & 9.200 & 10.000 \\
\hline \multirow{2}{*}{$6^{*}$} & $\mathrm{WT}_{6}$ & 5.566 & 5.167 & 5.567 & 4.833 & 6.700 & 7.767 & 6.700 & 7.500 \\
\hline & $\mathrm{DT}_{6}$ & 8.066 & 7.667 & 8.067 & 7.333 & 9.200 & 10.267 & 9.200 & 10.000 \\
\hline \multirow{2}{*}{7} & $\mathrm{WT}_{7}$ & 1.066 & 1.8 & 1.067 & 1.267 & 1.066 & 1.800 & 1.067 & 1.267 \\
\hline & $\mathrm{DT}_{7}$ & 2.066 & 2.8 & 2.067 & 2.267 & 2.066 & 2.800 & 2.067 & 2.267 \\
\hline \multirow{2}{*}{8} & $\mathrm{WT}_{8}$ & 4.433 & 4.9 & 4.433 & 4.634 & 1.700 & 2.300 & 1.700 & 2.034 \\
\hline & $\mathrm{DT}_{8}$ & 11.933 & 12.4 & 11.933 & 12.134 & 9.200 & 9.800 & 9.200 & 9.534 \\
\hline \multirow{2}{*}{$9^{\#}$} & $\mathrm{WT}_{9}$ & 3.767 & 3.434 & 3.766 & 4.300 & 2.967 & 2.967 & 2.966 & 1.700 \\
\hline & $\mathrm{DT}_{9}$ & 5.267 & 4.934 & 5.266 & 5.800 & 4.467 & 4.467 & 4.466 & 3.200 \\
\hline \multirow{2}{*}{$10^{\#}$} & $\mathrm{WT}_{10}$ & 4.800 & 4.467 & 4.800 & 2.934 & 1.867 & 1.867 & 1.867 & 2.467 \\
\hline & $\mathrm{DT}_{10}$ & 6.800 & 6.467 & 6.800 & 4.934 & 3.867 & 3.867 & 3.867 & 4.467 \\
\hline \multirow{2}{*}{$11^{\#}$} & $\mathrm{WT}_{11}$ & 5.133 & 4.800 & 5.134 & 4.466 & 4.333 & 2.200 & 4.334 & 1.866 \\
\hline & $\mathrm{DT}_{11}$ & 6.133 & 5.800 & 6.134 & 5.466 & 5.333 & 3.200 & 5.334 & 2.866 \\
\hline \multicolumn{2}{|r|}{ TWT } & 51.932 & 42.668 & 49.734 & 40.668 & 45.800 & 39.868 & 43.601 & 38.134 \\
\hline \multicolumn{2}{|r|}{ TDT } & 80.532 & 70.668 & 77.734 & 68.668 & 74.400 & 67.868 & 71.601 & 66.134 \\
\hline \multicolumn{2}{|r|}{ TTT } & 16.200 & 15.200 & 15.866 & 16.100 & 13.267 & 14.733 & 12.933 & 13.733 \\
\hline
\end{tabular}

Note. ${ }^{*}$ The freight operation site is shift-out site; ${ }^{\#}$ the freight operation site is shift-into site.

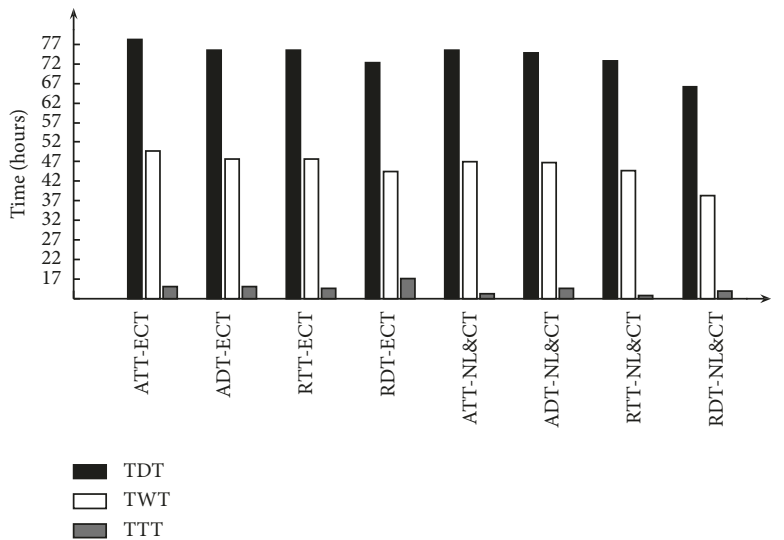

(a)

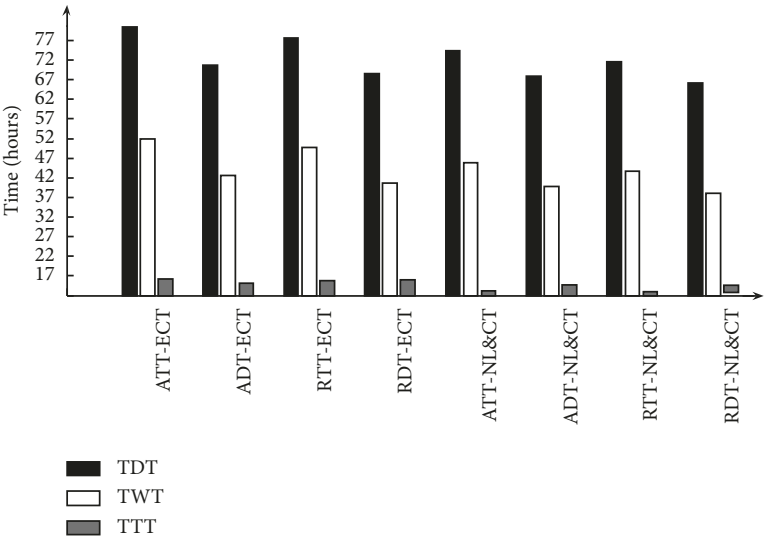

(b)

FIGURE 22: Results of comparison for different shunting strategies on PTWS-SSMS: result for different shunting strategies under (a) SSMECT and (b) SSM-NL\&CT.

\section{Conclusions}

In this paper, we discuss the placing-in and taking-out shunting strategies based on the branch-shaped freight operation network. We identify the decoupling-coupling local wagons process in a railway station, delivery the local wagons group process from the railway station to freight operation sites, and picking up the local wagons group process from the freight operation sites to railway station.
On this basis, PTWS is divided into decoupling-coupling wagons subsystem, placing-in local wagons subsystem, and taking-out local wagons subsystem. And then, the simulation platform encompassing two typical scenarios of PTWS is developed by Arena simulation software. Under scenarios of PTWS-SO, we provide the eight shunting strategies. Under scenarios of PTWS-SSMS, we present two different span-shift-moving strategies and generate sixteen hybrid shunting strategies. Computational experiments and 
concrete examples demonstrate that the simulation platform can implement an intuitive environment, assess the impacts produced by the adoption of different shunting strategies, and provide the efficient organization scheme of PTWS working for operators. Instead of obscure and inaccessible mathematical methodology, computer-based simulation platform can intuitively provide the decision-makers with the help they need in creating the strategies for development.

In our future work, prospective research may focus on extending the PTWS by introducing further realistic aspects and constraints. The consideration of more realistic aspects and constraints adds complexity to the problem of PTWS. In spite of this, we have shown that the simulation platform can handle very complex system and provide the efficient organization scheme.

\section{Data Availability}

The data used to support the findings of this study are included within the article.

\section{Conflicts of Interest}

The authors declare that there are no conflicts of interest regarding the publication of this paper.

\section{Acknowledgments}

This research was supported by the National Natural Science Foundation of China (Grant nos. U1604150 and U1804151). The support is gratefully acknowledged.

\section{References}

[1] L. Dahle, H. Andersson, M. Christiansen, and M. G. Speranza, "The pickup and delivery problem with time windows and occasional drivers," Computers \& Operations Research, vol. 109, pp. 122-133, 2019.

[2] S. Urrutia and D. De Werra, "What are the worst cases in constrained last-in-first-out pick-up and delivery problems?," European Journal of Operational Research, vol. 270, no. 2, pp. 430-434, 2018.

[3] T. Adamo, T. Bektaş, G. Ghiani, E. Guerriero, and E. Manni, "Path and speed optimization for conflict-free pickup and delivery under time windows," Transportation Science, vol. 52, no. 4, pp. 739-755, 2018.

[4] M. N. Haddad, R. Martinelli, T. Vidal et al., "Large neighborhood-based metaheuristic and branch-and-price for the pickup and delivery problem with split loads," European Journal of Operational Research, vol. 270, no. 3, pp. 1014-1027, 2018.

[5] N. Danloup, H. Allaoui, and G. Goncalves, "A comparison of two meta-heuristics for the pickup and delivery problem with transshipment," Computer \& Operations Research, vol. 100, pp. 155-171, 2019.

[6] J. Wang, Y. Yu, and J. Tang, "Compensation and profit distribution for cooperative green pickup and delivery problem," Transportation Research Part B: Methodological, vol. 113, pp. 54-69, 2018.

[7] L. Zhu and J.-B. Sheu, "Failure-specific cooperative recourse strategy for simultaneous pickup and delivery problem with stochastic demands," European Journal of Operational Research, vol. 271, no. 3, pp. 896-912, 2018.

[8] P. Györgyi and T. Kis, "A probabilistic approach to pickup and delivery problems with time window uncertainty," European Journal of Operational Research, vol. 274, no. 3, pp. 909-923, 2019.

[9] C. Li, L. Gong, Z. Luo, and A. Lim, "A branch-and-price-andcut algorithm for a pickup and delivery problem in retailing," Omega, vol. 89, pp. 71-91, 2019.

[10] V. Ghilas, J.-F. Cordeau, E. Demir, and T. V. Woensel, "Branch-and-price for the pickup and delivery problem with time windows and scheduled lines," Transportation Science, vol. 52, no. 5, pp. 1191-1210, 2018.

[11] T. Capelle, C. E. Cortés, M. Gendreau, P. A. Rey, and L.-M. Rousseau, "A column generation approach for locationrouting problems with pickup and delivery," European Journal of Operational Research, vol. 272, no. 1, pp. 121-131, 2019.

[12] E. H. C. Lu and Y. W. Yang, "A hybrid route planning approach for logistics with pickup and delivery," Expert Systems with Applications, vol. 118, pp. 482-492, 2019.

[13] B. Li, H. Xuan, X. Yang, and X. Wang, "A novel parameterised approximation approach based on bi-level programming for integration transport scheduling problem," Journal of the Operational Research Society, vol. 70, no. 2, pp. 212-225, 2019.

[14] B. Li, X. Yang, and H. Xuan, "A hybrid simulated annealing heuristic for multistage heterogeneous fleet scheduling with fleet sizing decisions," Journal of Advanced Transportation, vol. 2019, Article ID 5364201, 19 pages, 2019.

[15] L. D. Giovanni, N. Gastaldon, and F. Sottovia, "A two-level local search heuristic for pickup and delivery problems in express freight trucking," Networks, vol. 74, no. 4, pp. 333350, 2019.

[16] D. Goeke, "Granular tabu search for the pickup and delivery problem with time windows and electric vehicles," European Journal of Operational Research, vol. 278, no. 3, pp. 821-836, 2019.

[17] A. G. Dragomir, D. Nicola, A. Soriano, and M. Gansterer, "Multidepot pickup and delivery problems in multiple regions: a typology and integrated model," International Transactions in Operational Research, vol. 25, no. 2, pp. 569-597, 2017.

[18] Y. Wang and F. Chen, "Packed parts delivery problem of automotive inbound logistics with a supplier park," Computers \& Operations Research, vol. 101, pp. 116-129, 2019.

[19] M. W. Ulmer, B. W. Thomas, and D. C. Mattfeld, "Preemptive depot returns for dynamic same-day delivery," EURO Journal on Transportation and Logistics, vol. 8, no. 4, pp. 327-361, 2018.

[20] E. Benavent, M. Landete, J.-J. Salazar-González, and G. Tirado, "The probabilistic pickup-and-delivery travelling salesman problem," Expert Systems With Applications, vol. 121, pp. 313-323, 2019.

[21] A. Bettinelli, V. Cacchiani, T. G. Crainic, and D. Vigo, "A branch-and-cut-and-price algorithm for the multi-trip separate pickup and delivery problem with time windows at customers and facilities," European Journal of Operational Research, vol. 279, no. 3, pp. 824-839, 2019.

[22] A. M. Alyasiry, M. Forbes, and M. Bulmer, "An exact algorithm for the pickup and delivery problem with time windows and last-in-first-out loading," Transportation Science, vol. 53, no. 6, pp. 1695-1705, 2019.

[23] M. Mahmoudi, J. Chen, T. Shi, Y. Zhang, and X. Zhou, “A cumulative service state representation for the pickup and 
delivery problem with transfers," Transportation Research Part B: Methodological, vol. 129, pp. 351-380, 2019.

[24] Z. Huang, W. Huang, and F. Guo, "Integrated sustainable planning of self-pickup and door-to-door delivery service with multi-type stations," Computers \& Industrial Engineering, vol. 135, pp. 412-425, 2019.

[25] F. Jaehn, A. Otto, and K. Seifried, "Shunting operations at flat yards: retrieving freight railcars from storage tracks," $O R$ Spectrum, vol. 40, no. 2, pp. 367-393, 2017.

[26] L.-M. Kieu, D. Ngoduy, N. Malleson, and E. Chung, "A stochastic schedule-following simulation model of bus routes," Transportmetrica B: Transport Dynamics, vol. 7, no. 1, pp. 1588-1610, 2019.

[27] B. F. Zhu, Q. Zhou, and Y. Tian, "Simulation-based quantitative evaluation method for water-water transshipment coal terminals," Ournal of Waterwagy Port Coastal and Ocean Engineering, vol. 145, no. 3, p. 14, 2019.

[28] X. Liu, L. Li, X. Liu, and T. Zhang, "Analysis of passenger flow and its influences on HVAC systems: an agent based simulation in a Chinese hub airport terminal," Building and Environment, vol. 154, pp. 55-67, 2019.

[29] C. Archetti, M. G. Speranza, and D. Weyland, "A simulation study of an on-demand transportation system," International Transactions in Operational Research, vol. 25, no. 4, pp. 1137-1161, 2018.

[30] S. Lam, J. Taghia, and J. Katupitiya, "Evaluation of a transportation system employing autonomous vehicles," Journal of Advanced Transportation, vol. 50, no. 8, pp. 2266-2287, 2019.

[31] M. Deja, M. Dobrzyński, M. S. Wiśniewska, and A. Wisniewska, "Simulation studies into quayside transport and storage yard operations in container terminals," Polish Maritime Research, vol. 24, no. s1, pp. 46-52, 2017.

[32] R. Florin, P. Mihaela, R. Eugen, R. Aura, R. Mircea, and D. Oana, "Assessing the transit capacity of port shunting yards through discrete simulation," Transport Problems, vol. 14, no. 4, pp. 101-111, 2019. 


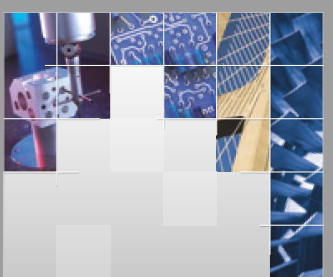

\section{Enfincering}
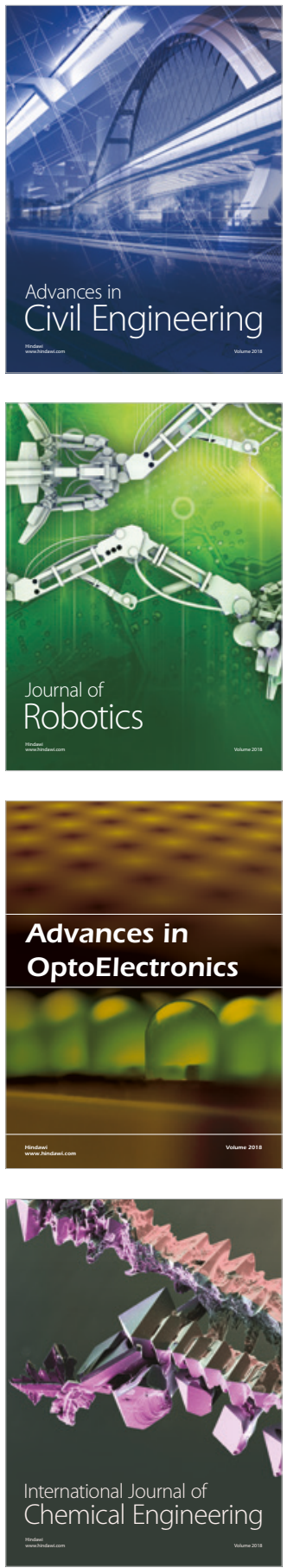

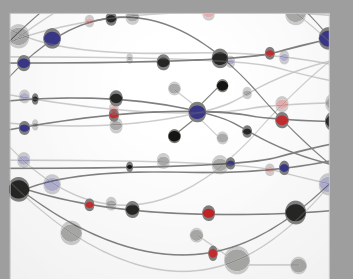

\section{Rotating \\ Machinery}

The Scientific World Journal

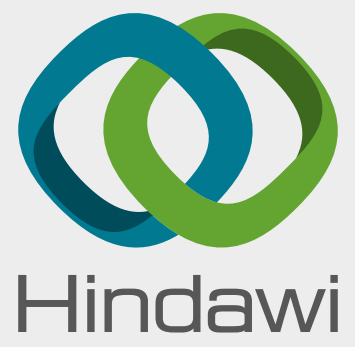

Submit your manuscripts at

www.hindawi.com
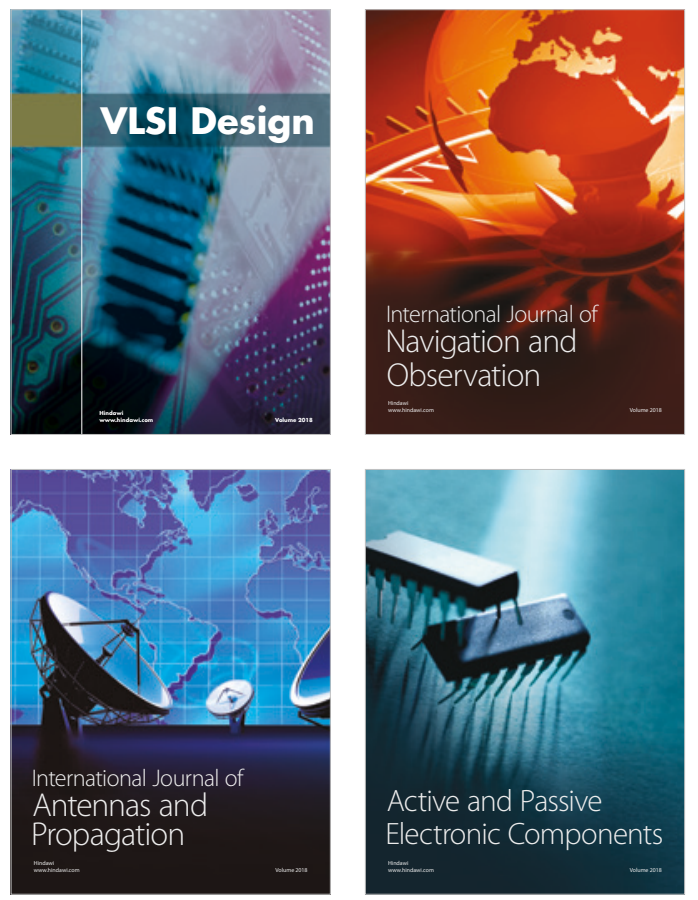
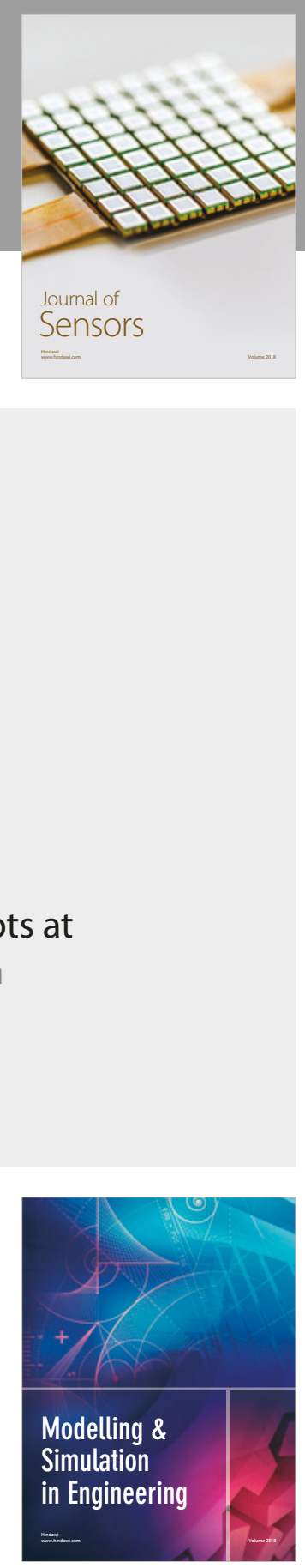

\section{Advances \\ Multimedia}
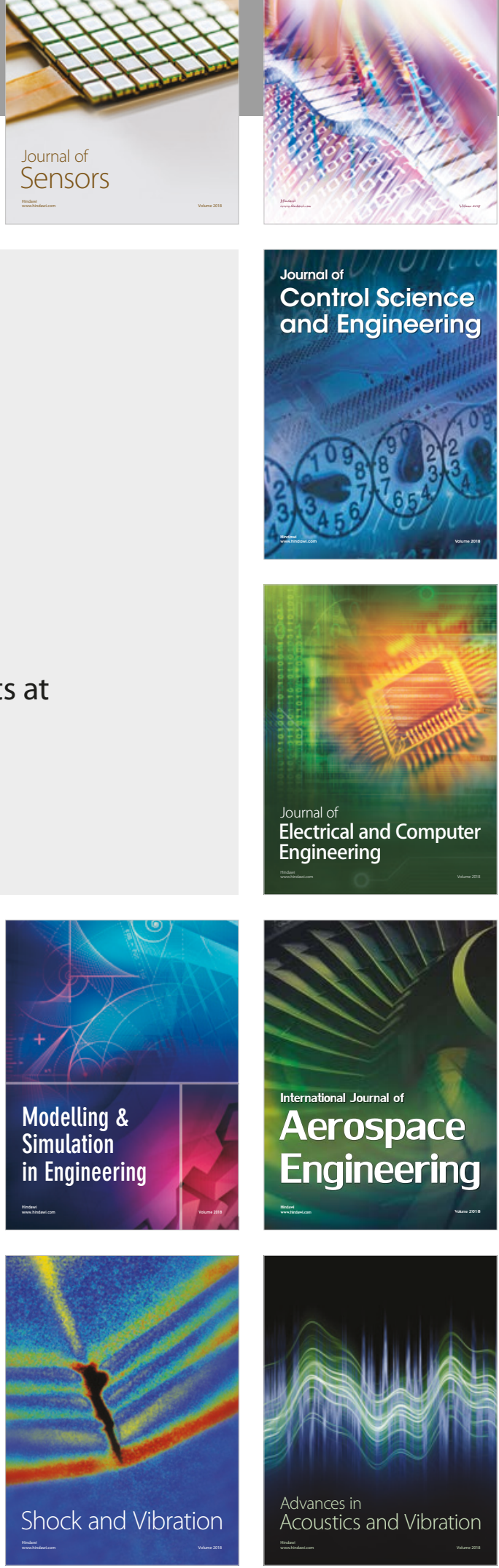